ARTICLE

DOI: $10.1038 / s 41467-017-01974-7$

\title{
Identifying the ubiquitination targets of E6AP by orthogonal ubiquitin transfer
}

Yiyang Wang (1) 1, Xianpeng Liu ${ }^{2}$, Li Zhou', Duc Duong ${ }^{3}$, Karan Bhuripanyo ${ }^{1,4}$, Bo Zhao ${ }^{5}$, Han Zhou', Ruochuan Liu', Yingtao Bi ${ }^{6}$, Hiroaki Kiyokawa ${ }^{2} \&$ Jun Yin ${ }^{1}$

E3 ubiquitin (UB) ligases are the ending modules of the E1-E2-E3 cascades that transfer UB to cellular proteins and regulate their biological functions. Identifying the substrates of an E3 holds the key to elucidate its role in cell regulation. Here, we construct an orthogonal UB transfer (OUT) cascade to identify the substrates of E6AP, a HECT E3 also known as Ube3a that is implicated in cancer and neurodevelopmental disorders. We use yeast cell surface display to engineer E6AP to exclusively transfer an affinity-tagged $U B$ variant ( $x U B$ ) to its substrate proteins. Proteomic identification of $x$ UB-conjugated proteins in HEK293 cells affords 130 potential E6AP targets. Among them, we verify that MAPK1, CDK1, CDK4, PRMT5, $\beta$-catenin, and UbxD8 are directly ubiquitinated by E6AP in vitro and in the cell. Our work establishes OUT as an efficient platform to profile E3 substrates and reveal the cellular circuits mediated by the E3 enzymes.

\footnotetext{
${ }^{1}$ Department of Chemistry, Center for Diagnostics \& Therapeutics, Georgia State University, Atlanta, GA 30303, USA. ${ }^{2}$ Department of Pharmacology, Northwestern University, Chicago, IL 60611, USA. ${ }^{3}$ Integrated Proteomics Core, Emory University, Atlanta, GA 30322, USA. ${ }^{4}$ Department of Chemistry, University of Chicago, Chicago, IL 60637, USA. ${ }^{5}$ Engineering Research Center of Cell \& Therapeutic Antibody, Ministry of Education, and School of Pharmacy, Shanghai Jiao Tong University, Shanghai, 200240, China. ${ }^{6}$ Department of Preventive Medicine, Northwestern University, Chicago, IL 60611, USA. Correspondence and requests for materials should be addressed to H.K. (email: kiyokawa@northwestern.edu) or to J.Y. (email: junyin@gsu.edu)
} 
biquitin (UB), a 76-residue protein riding on a E1-E2-E3 enzymatic cascade, is a key messenger in cell signaling ${ }^{1}$. UB attachment to cellular proteins regulates many key processes such as protein degradation, subcellular trafficking, enzymatic turnover, and complex formation. E1 activates UB with the formation of a thioester linkage between a catalytic Cys of $\mathrm{E} 1$ and the C-terminal Gly of $\mathrm{UB}^{2}$. UB bound to E1 is loaded on an E2 in a thioester exchange reaction to form a UB E2 conjugate (" $\sim$ " designates the thioester bond) ${ }^{3}$. E2 then carries UB to an E3 that recruits target proteins for UB conjugation ${ }^{4-6}$. The human genome encodes $2 \mathrm{E} 1 \mathrm{~s}$, at least $40 \mathrm{E} 2 \mathrm{~s}$ and more than 600 $\mathrm{E} 3 \mathrm{~s}^{3}, 7,8$. Since E3s recognize protein ubiquitination targets, they often play key regulatory roles, and their malfunction drives the development of many diseases including cancer, neurodegeneration, and inflammation ${ }^{9,10}$. For example, E6AP, also known as Ube3a, is a E3 with a signature HECT domain for E2 binding ${ }^{11}$. E6AP is a critical regulator of neuron development; loss of its activity results in Angelman syndrome (AS), and duplications of chromosomal region 15q11-13 including its encoding gene Ube $3 a$ are associated with autism spectrum disorders (ASD) ${ }^{12-15}$. E6AP promotes tumorigenesis upon infection of high-risk human papillomavirus-it forms a complex with the viral oncoprotein E6 to ubiquitinate p53 and induce its degradation ${ }^{11,16}$. Other non-HECT E3s may bind the E2 UB conjugate through a Ring, Ring-between-Ring (RBR) or U-box motif ${ }^{4,6,7}$. Regardless of the type of interactions with E2s, an E3 may uptake UB from multiple $\mathrm{E} 2 \mathrm{~s}$, and various E3s transfer UB to an overlapping pool of substrates. The complex cross-reactivities among E2, E3, and substrates make it a significant challenge to profile the substrates of a specific E3 to map it on the cell signaling network.

We envision an "orthogonal UB transfer (OUT)" pathway in which a UB variant (xUB) is confined to a single track of engineered $\mathrm{xE} 1, \mathrm{xE} 2$, and $\mathrm{xE} 3$ would guide the transfer of $\mathrm{xUB}$ exclusively to the substrate of a specific E3 (" $x$ " designates engineered UB or enzyme variants orthogonal to their native partners $)^{17}$. By expressing $\mathrm{xUB}$ and the OUT cascade of $\mathrm{xE} 1-\mathrm{xE} 2-\mathrm{xE} 3$ in the cell and purifying cellular proteins conjugated to $\mathrm{xUB}$, we would be able to identify the direct substrates of an E3. The development of the OUT cascade removes the cross-reacting paths among various E2s and E3s. It enables the assignment of E3 substrates by directly following xUB transfer through the E3 instead of reading some indirect indicators of protein ubiquitination such as affinity binding with E3, or change of protein stability or ubiquitination levels upon E3 expression.

To implement OUT, we need to engineer orthogonal pairs of $\mathrm{xUB}-\mathrm{xE} 1, \mathrm{xE} 1-\mathrm{xE2}$, and $\mathrm{xE2}-\mathrm{xE} 3$ that are free of crossreactivities with native $\mathrm{E} 1, \mathrm{E} 2$, and $\mathrm{E} 3$ to secure the exclusive transfer of $\mathrm{xUB}$ to the substrates of an E3 in the cell. We previously reported engineering orthogonal $\mathrm{xUB}-\mathrm{xE} 1$ and $\mathrm{xE} 1-\mathrm{xE} 2$ pairs by phage display ${ }^{17}$. We also generated the $\mathrm{xUB}-\mathrm{xE} 1$ pairs with the two human E1, Uba1, and Uba6, respectively, to differentiate their targets of UB transfer in the cell ${ }^{18}$. Here we report that we have accomplished the last leg of OUT engineering: we used yeast cell surface display to engineer an orthogonal $\mathrm{xE2}-\mathrm{xE} 3$ pair with the HECT E3 E6AP; we expressed the OUT cascade in HEK293 cells to profile E6AP substrates; and we identified a number of key signaling proteins as E6AP substrates and established regulatory circuits mediated by UB transfer through E6AP.

\section{Results}

Constructing the xUB-xUbal and the xUba1-xUbcH7 pair. We previously generated an $\mathrm{xUB}-\mathrm{xE} 1$ pair with the E1 enzyme Uba1 from $S$. cerevisiae ${ }^{17}$. Using phage selection, we found that the two mutations in $\mathrm{xUB}$ (R42E and R72E) would block $\mathrm{xUB}$ recognition by wt Uba1, yet by incorporating mutations Q576R, S589R and
D591R into the adenylation domain of yeast Uba1, we could restore the activity of $\mathrm{xUB}$ with $\mathrm{E} 1$ to form $\mathrm{xUB} \sim \mathrm{E} 1$ thioester conjugates (Supplementary Fig. 1a, b). We also introduced mutations E1004K, D1014K and E1016K into the UFD domain of the yeast Ubal to block its interaction with the wt E2s (Supplementary Fig. 1c). We then used phage display to identify mutations in the $\mathrm{N}$-terminal helix of the Ubc1, a yeast E2, to restore E1-E2 interaction and enable UB transfer to the E2 enzyme. By combining the mutations in the adenylation and the UFD domains of yeast Uba1, we generated the E1 mutant xUbal that can specifically transfer $\mathrm{xUB}$ to $\mathrm{xUbc1}$, the E2 mutant from phage selection (Table 1). In contrast, $\mathrm{xUB}$ cannot be activated by wt Ubal for its transfer to wt E2s. xUbal cannot activate wt UB, neither can it transfer $x U B$ to wt E2. Thus, the $x U B-x E 1$ and $\mathrm{xE} 1-\mathrm{xE} 2$ pairs are orthogonal to their native partners, and they can assemble a two-step cascade to transfer $\mathrm{xUB}$ to a designated E2.

Our success in engineering the $\mathrm{xUB}-\mathrm{xE} 1$ and $\mathrm{xE} 1-\mathrm{xE} 2$ pairs with the yeast system is instrumental for constructing the OUT cascade in the human cell. We found $\mathrm{xUB}$ is not catalytically active with either of the human E1s, Ubal (also known as Ube1), or $\mathrm{Uba}^{18}$. Since Ubal plays a major role in supporting protein ubiquitination in the human cells ${ }^{8}$, we decided to engineer human Ubal as the xE1 for the OUT cascade. Based on the sequence homology between the human and yeast Uba1, we identified residues Q608, S621 and D623 in the adenylation domain and E1037, D1047, and E1049 in the UFD domain of human Ubal matching the sites of mutations in the yeast Ubal (Supplementary Fig. 1d, e). We mutated these residues to R or K of the opposite charge to generate human xUbal (Table 1). As expected, we found human xUbal is reactive with $\mathrm{xUB}$ by forming $\mathrm{xUB} \sim \mathrm{xUba1}$ conjugate, yet it rejects wt $\mathrm{UB}$ in the activation reaction (Fig. 1a). Moreover, xUbal cannot transfer $\mathrm{xUB}$ to wt human $\mathrm{E} 2 \mathrm{~s}$ such as $\mathrm{UbcH} 7$ due to the mutations in the UFD domain of Ubal.

To restore $\mathrm{xUB}$ transfer to $\mathrm{E} 2 \mathrm{~s}$, we generated orthogonal $\mathrm{xE} 1-$ $\mathrm{xE} 2$ pairs based on the sequence homology between the yeast and human E2s. The N-terminal helix of E2 plays a key role in binding the UFD domain of the E1 as shown in the crystal structures of yeast $S$. pombe Uba1 in complex with E2 Ubc4, and the modeled structure of $S$. cerevisiae Uba1 bound with Ubc1 (Supplementary Fig. 1a, c) ${ }^{19,20}$. The sequences of the N-terminal helices of E2s from yeast and human align well with highly conserved $\mathrm{K}$ or $\mathrm{R}$ residues at positions 5, 6, and 9 ( $\mathrm{UbcH} 7$ numbering) (Supplementary Fig. 1f). Based on the sequence alignment, we mutated $\mathrm{R} 5$ and $\mathrm{K} 9$ in $\mathrm{UbcH} 7$ to Glu following the mutations in yeast $\mathrm{xUbcl}$ and found the newly constructed $\mathrm{xUbcH7}$ can pair with xUba1 to accept xUB transfer (Fig. 1a and Table 1). We have thus constructed an xUba1-xUbcH7 pair for $\mathrm{xUB}$ transfer through the OUT cascade. Since UbcH7 partners with HECT E3 in the cell, the exclusive delivery of xUB by xUba1

\begin{tabular}{|c|c|}
\hline $\begin{array}{l}x \cup B \text { (human) } \\
x E 1\end{array}$ & R42E, R72E \\
\hline xUba1 (yeast) & Q576R, S589R, D591R, E1004K, D1014K, E1016K \\
\hline $\begin{array}{l}x \text { XUba1 (human) } \\
x E 2\end{array}$ & Q608R, S621R，D623R，E1037K， D1047K，E1049K \\
\hline $\begin{array}{l}x U b c 1 \text { (yeast) } \\
x U b c H 7 \text { (human) } \\
x E 3\end{array}$ & $\begin{array}{l}\text { K5D, R6E, K9E, E10Q, Q12L } \\
\text { R5E, K9E }\end{array}$ \\
\hline xE6AP (YW6) & D651R, D652E, M653W, M654H \\
\hline
\end{tabular}


a

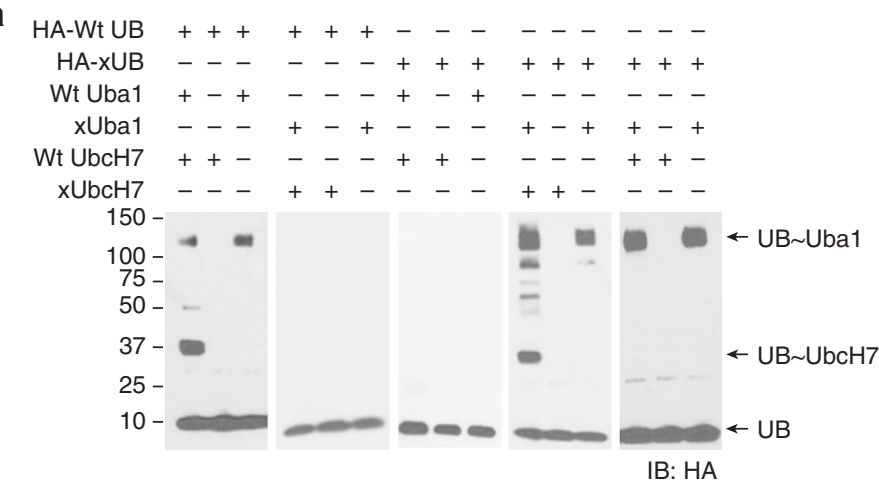

b

HA-Wt UB +++---
HA-xUB ---+++
Wt Uba1 ++----
xUba1 ---++-
Wt UbcH7 +-+---
xUbcH7 ---+-+
6AP-HECT ++++++

\section{C}

HA-Wt UB +++--HA-xUB - - + + + Wt Uba1 ++--xUba1 - - + + -

Wt UbcH7 + - + - $\mathrm{xUbcH} 7--++$ Flag-E6AP-HECT ++++++ Flag-Nedd4-HECT ++++++
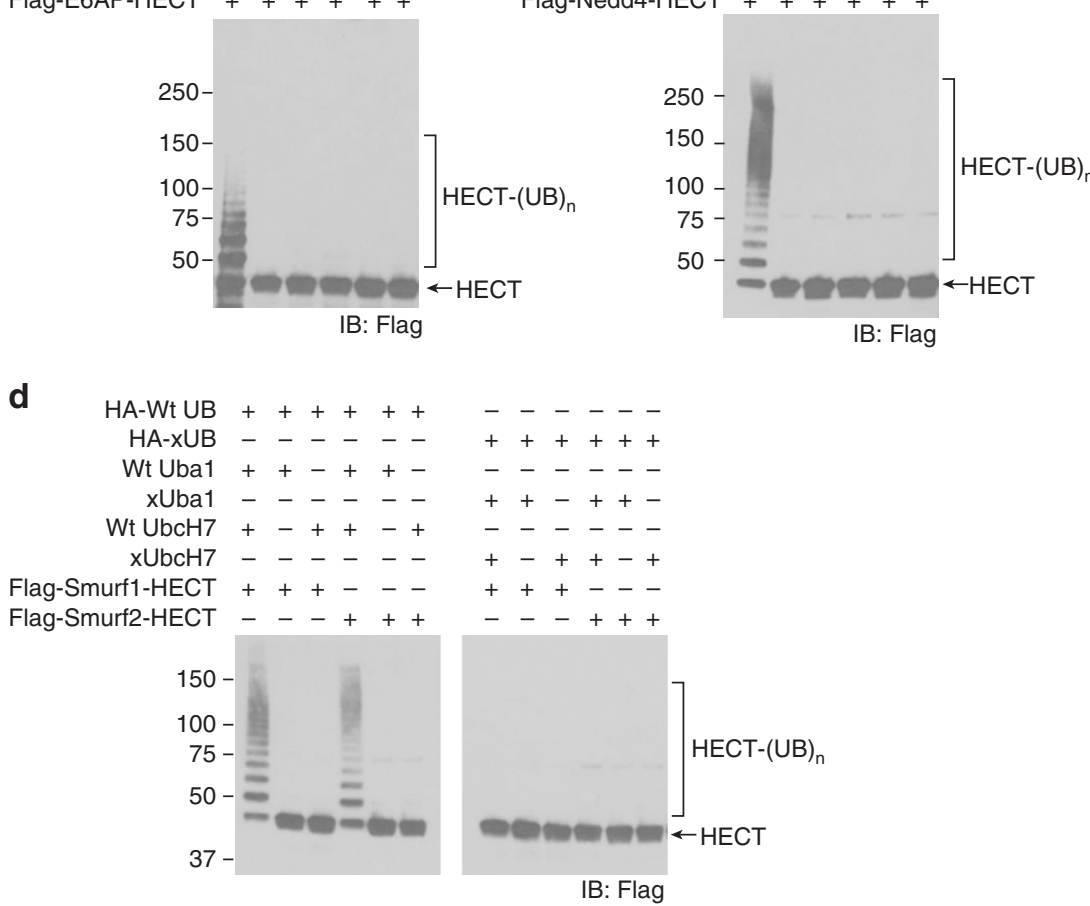

Fig. 1 Orthogonality of the $x U b a 1-x U b c H 7$ pair with the wt UB transferring enzymes. a Wt UB can be transferred to wt UbcH7 by wt human Uba1, but it cannot be activated by human $x$ Uba1 for transferring to $x U b c H 7$. Vice versa, $x U B$ can be activated by $x U b a 1$ for transferring to $x U b c H 7$, but it cannot be activated by wt Uba1 for transferring to $w \mathrm{UbcH7}$, nor can it be transferred to wt $\mathrm{UbcH7}$ by $\mathrm{xUba1}$. The protein gels were run under non-reducing conditions to preserve the thioester conjugates of UB E1 and UB E2. b-d HECT domains of wt E6AP, Nedd4, Smurf1, and Smurf2 can be loaded with wt UB

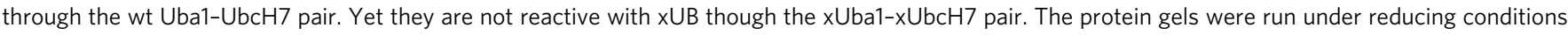
to probe the auto-ubiquitination of the HECT enzymes

to $\mathrm{xUbcH7}$ paved the way for transferring $\mathrm{xUB}$ to a specific HECT E3 to profile its substrate proteins.

Constructing the $x$ UbcH7-xHECT pair with E6AP. The Nterminal helix of $\mathrm{UbcH} 7$ is a key element not only for interaction with E1s but also for interaction with E3s (Fig. 2a, b). We found the R5E and $\mathrm{K} 9 \mathrm{E}$ mutants in the N-terminal helix of $\mathrm{xUbcH7}$ interfered with the transfer of xUB to wt HECT E3s such as E6AP, Nedd4, Smurf1, and Smurf2 (Fig. 1b-d). This is advantageous for the construction of the OUT cascade since it is preferred that $\mathrm{xE} 2$ would not pair with wt E3s to randomly transfer $\mathrm{xUB}$ to any E3 substrates in the cell. Our goal was to bridge $\mathrm{xUB}$ transfer through the last step of the OUT cascade by engineering an orthogonal $x U b c H 7-x E 6 A P$ pair. For this purpose, we used yeast cell surface display to select for HECT mutants of E6AP that would restore binding with $\mathrm{xUbcH7}$ to enable $\mathrm{xUB}$ loading on the HECT domain (Fig. 2c). For yeast selection, a HECT library of E6AP was expressed as fusions to the yeast cell surface protein Aga2P with each yeast cell displaying a specific member of the HECT library $^{21}$. The yeast library was then reacted with biotinlabeled xUB, xUba1, and xUbcH7. HECT mutants catalytically active with $\mathrm{xUbcH7}$ were loaded with $\mathrm{xUB}$ through the formation of $\mathrm{xUB} \sim \mathrm{HECT}$ thioester conjugate. The catalytically active HECT mutants were further auto-ubiquitinated by xUB through Lys modification. As a result, the corresponding yeast cells were labeled with biotin that would bind to streptavidin conjugated 

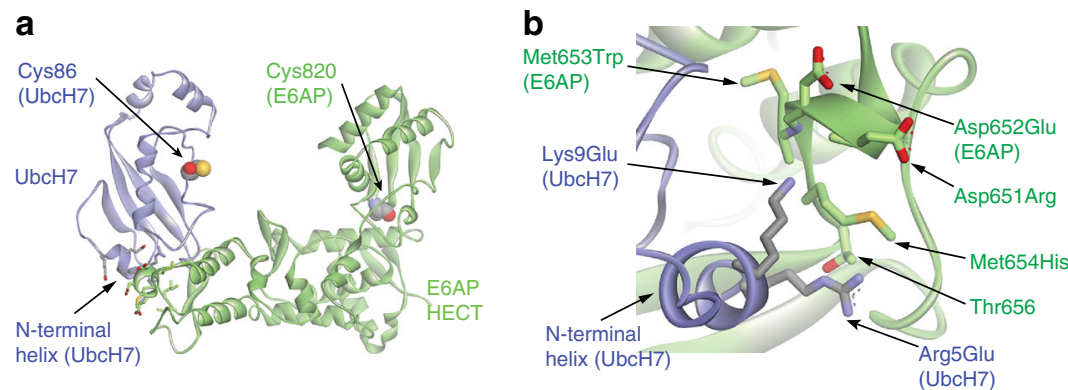

d
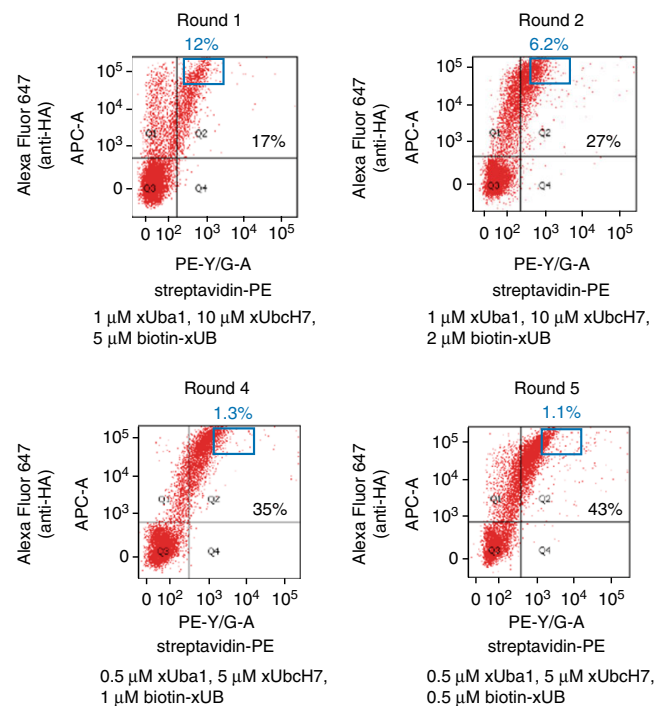

\section{C}

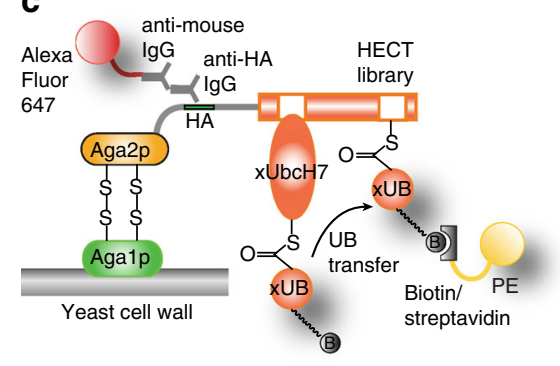

e

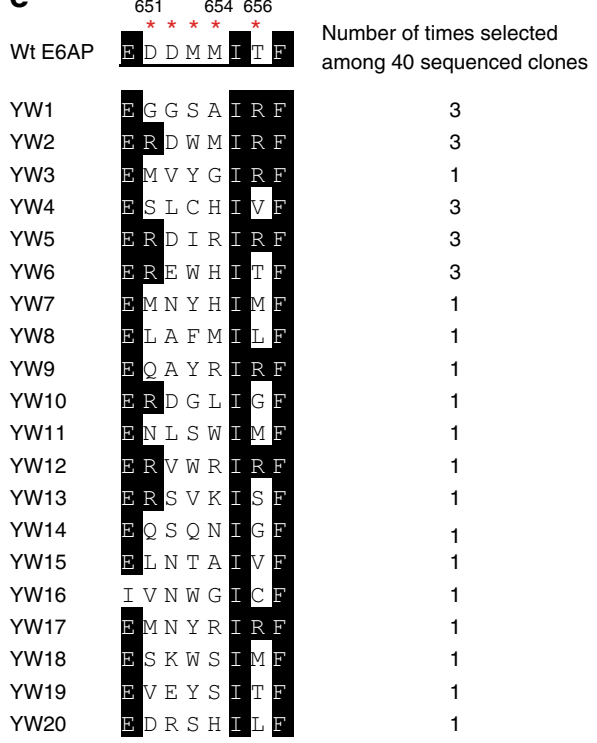

Fig. 2 Yeast selection of E6AP library to engineer the xUbcH7-xE6AP pair. a Crystal structure of the E6AP HECT domain in complex with UbcH7 (PDB ID $1 \mathrm{C} 4 \mathrm{Z})^{22}$. The $\mathrm{N}$-terminal helix of $\mathrm{UbcH7}$ plays a key role in interacting with the HECT domain. The catalytic Cys residues in UbcH7 and the E6AP HECT are shown in CPK models. b Detailed interactions between the N-terminal helix of UbcH7 and the HECT domain of E6AP. To complement the R5E and K9E mutations in $\mathrm{xUbcH7}$, D651, D652, M653, M654 and T656 in the HECT domain of E6AP were randomized for library selection by yeast cell surface display. HECT mutant YW6 with mutations D651R, D652E, M653W, and M654H was selected and used as xHECT in this study. c Yeast cell surface display of the HECT domain of E6AP and selection of the HECT library based on the transfer of biotin-xUB from xUbcH7 to the HECT domain. Biotin-xUB attached to HECT was labeled with streptavidin-PE, and the HA tag fused to the N-terminus of the HECT domain was labeled with a mouse anti-HA antibody and an anti-mouse IgG conjugated with Alexa 647. d FACS sorting of the HECT library of E6AP to select for yeast cells (EBY100) doubly labeled with PE and Alexa 647 as indicators of biotin-xUB conjugation and display of HECT on the cell surface, respectively. The HECT library underwent six rounds of xUB loading, streptavidin and antibody labeling, and cell sorting. Percentages in black designate fraction of yeast cells doubly labeled with PE and Alexa 647. The frames and percentages in blue designate the fraction of yeast cells collected by FACS in each round of selection. e Sequence alignment of the E6AP HECT clones selected by yeast cell surface display. Residues denoted by red stars were randomized in the HECT library

with phycoerythrin (PE). For selection of yeast cells displaying the HECT domain, a mouse anti-HA antibody was used to bind to the HA tag at the $\mathrm{N}$ terminus of the HECT and it was subsequently labeled with an anti-mouse IgG conjugated with Alexa 647. Fluorescence-activated cell sorting (FACS) was used to enrich the cells that were double labeled with the PE and Alexa 647. In this way, the sorting was to enrich cells that displayed catalytically active HECT domains capable of bridging xUB transfer from $\mathrm{xUbcH7}$. We validated the selection protocol by displaying the wt HECT domain of E6AP on the yeast cell surface, and demonstrating the efficient labeling of the yeast cells by biotin-wt UB transferred through the wt Ubal-UbcH7 pair (Supplementary Fig. 2).

We constructed a HECT library of E6AP based on the crystal structure of the HECT domain with UbcH7 (Fig. 2a, b $)^{22}$. Residues R5 and K9 in the N-terminal helix of UbcH7 were mutated to Glu in $\mathrm{xUbcH7}$ and the crystal structure shows that these residues mainly interact with a helical turn in the HECT domain of E6AP. R5 of UbcH7 is in close distance (4.6 $\AA$ ) with the hydroxyl group of T656 of HECT. It also packs on the side chain of M654 that is $3.2 \AA$ away. K9 of UbcH7 may form salt bridges with HECT D651 and D652 that are a short distance apart $(4.5 \AA)$. We thus decided to randomize E6AP residues D651, D652, M653, M654, and T656 to generate the HECT library.

We expressed the library on the yeast cell surface and carried out the selection by transferring biotin-xUB to the HECT mutants through the xUba1-xUbcH7 pair. Cells were labeled with streptavidin and antibody conjugates with fluorophores as in the model selection, and FACS was performed to harvest cells that were double labeled with PE and Alexa 647. Cells collected were cultured for next round of biotin-xUB loading, fluorescent labeling, and FACS. After 6 rounds of sorting, 51\% of the cells were double labeled with both fluorophores suggesting a population of HECT mutants with efficient xUB transfer activity from $\mathrm{xUbcH7}$ were selected (Fig. 2d). DNA sequencing of the 40 clones from the $6^{\text {th }}$ round of sorting showed a clear pattern of convergence (Fig. 2e). Clones appearing multiple times tend to 
a

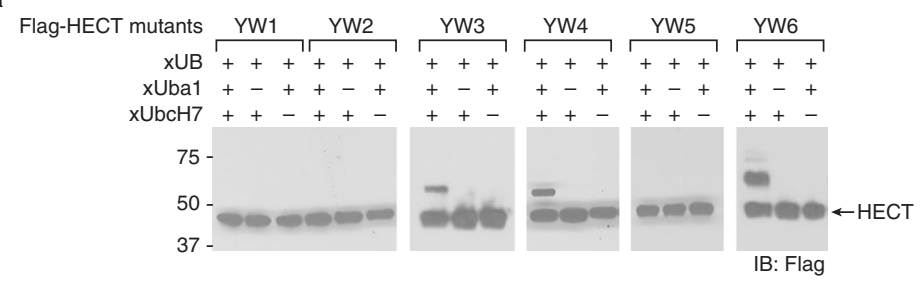

b
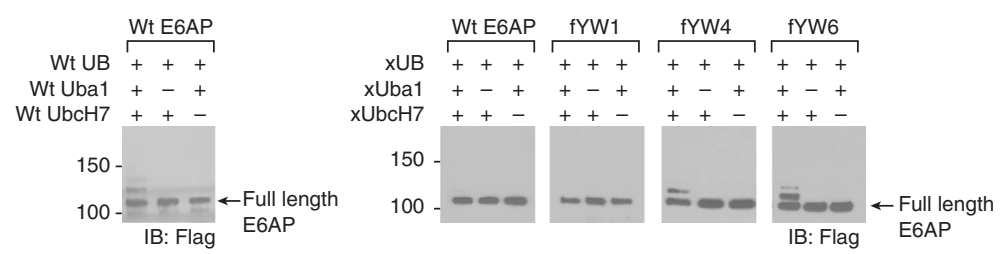

C
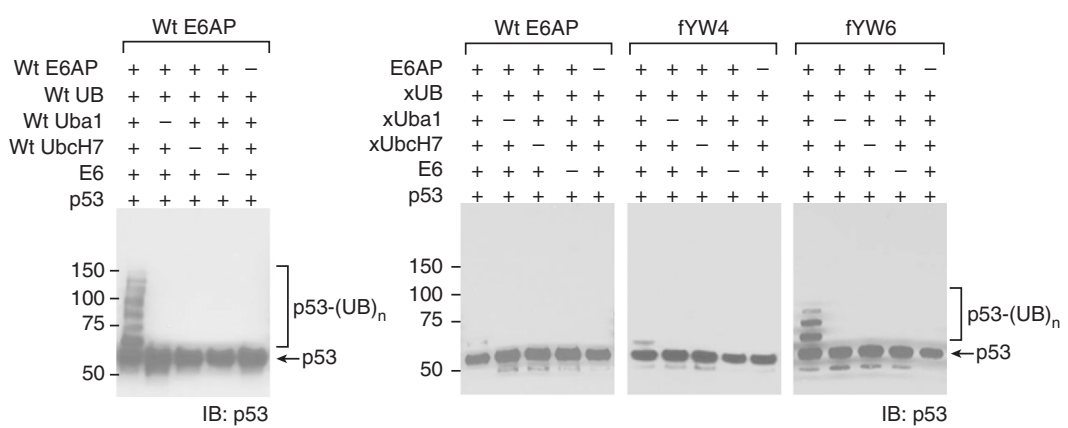

Fig. 3 Activity in $x U B$ transfer by the E6AP mutants identified from yeast cell selection. a Activity of the HECT mutants YW1-6 in auto-ubiquitination by $x U B$ through the $x U b a 1-x U b c H 7$ pair. b Activity of the full-length E6AP mutants $f Y W 1, f Y W 4$, and fYW6 in auto-ubiquitination by the $x U b a 1-x U b c H 7$ pair (right panel). While wt E6AP can be auto-ubiquitinated by the wt Uba1-UbcH7 pair (left panel), it cannot be conjugated to xUB by the xUba1-xUbcH7 pair (right panel). c p53 ubiquitination by wt E6AP and fYW4 and fYW6 in the presence of E6. p53 can be efficiently ubiquitinated by wt UB through the wt Uba1-UbcH7-E6AP cascade in the presence of E6 (left panel). p53 can also be efficiently modified by xUB through the engineered cascade of xUba1-xUbcH7-fYW6 in the presence of E6 (right panels). In contrast, xUB has very low activity in ubiquitinating p53 through the crossover cascade of $x U b a 1-x U b c H 7-w t ~ E 6 A P$ in the presence of E6. All blots are representative of at least three independent experiments

have D651 in HECT replaced with an Arg (YW2, YW5, and YW6). This change matches the charge reverse mutation of K9E in xUbcH7 (Fig. 2b). D652 of HECT, although randomized in the library, was most often unchanged or replaced with a similar Glu in the selected clones (YW2, YW5, and YW6). M653 is often replaced by aromatic residues such as Tyr and Trp in the selected clones (YW2, YW3, and YW6). M654 is replaced by positively charged Arg or His residues (YW4, YW5 and YW6), and residues selected at T656 is also quite converged showing a preference for positively charged Arg (YW1-3 and YW5). These changes match well with the charge reversal of R5E mutation in $\mathrm{xUbcH7}$. We thus assayed if the individual HECT mutants from FACS could mediate xUB transfer with the $\mathrm{xUba} 1-\mathrm{xUbcH} 7$ pair.

Verifying xUB transfer through E6AP mutants. We separately cultured yeast clones YW1-6 and reacted the yeast cells with the xUba1-xUbcH7 pair for biotin-xUB loading (Supplementary Fig. 3). We found yeast clones YW4 and YW6 had the strongest loading of biotin-xUB with $25 \%$ and $34 \%$ of the cells doubly labeled. To check the activities of individual HECT domains, we expressed mutants YW1-6 in E. coli and found YW3, YW4, and YW6 could be efficiently auto-ubiquitinated with XUB through the xUba1-xUbcH7 pair while YW1, YW2, and YW5 were not active for xUB transfer (Fig. 3a). We suspect the difference in activities of the HECT domains anchored on yeast cell surface and free in solution may be due to the change in their folding status in different environments. We replaced the wt HECT domain in E6AP with the mutant HECT of YW1, YW4, and YW6 to generate the full-length E6AP mutants FYW1, fYW4, and fYW6. We found fYW4 and fYW6 can be auto-ubiquitinated by $\mathrm{xUB}$ through the xUba1-xUbcH7 cascade (Fig. 3b). However, wt E6AP and fYW1 were not active in auto-ubiquitination by $x U B$ in combination with the xUba1-xUbcH7 pair. We then tested the transfer of $x \mathrm{UB}$ from the E6AP mutants to p53, a key ubiquitination target recruited by $\mathrm{E}^{11}{ }^{11}$. We found fYW6 could efficiently ubiquitinate p53 with xUB through the xUba1-xUbcH7-fYW6 cascade and the ubiquitination was dependent on the E6 protein (Fig. 3c). The activity of xUB transfer to p53 through fYW6 was approaching the activity of wt UB transfer through wt E6AP. In contrast, when the xUba1-xUbcH7 pair was reacted with wt E6AP, we only observed very low activity in transferring $\mathrm{xUB}$ to $\mathrm{p} 53$ suggesting the minimal cross-reactivity of $x U B$ with native UB transfer pathways (Fig. 3c). Comparing to fYW6, fYW4 was less active in transferring xUB to p53. We thus decided to use fYW6 as xE6AP in the OUT cascade to identify E6AP substrates in the cell (Table 1).

Verifying the orthogonality of OUT cascade in cells. We next tested if $\mathrm{xUB}$ could be exclusively transferred through the OUT cascade of xUba1-xUbcH7-xE6AP in the cells without crossing over to the wt UB transfer cascades (Fig. 4a). We constructed a lentiviral vector to express wt UB or xUB with tandem $6 \times$ His and biotin tags at the $\mathrm{N}$ terminus of UB (HBT-wt UB and HBT-xUB) ${ }^{23}$. We also screened HEK293 cells that stably expressed wt Ubal or xUba1 with an N-terminal Flag tag. Transient expression of HBTwt UB and HBT-xUB in these cells followed by affinity pull-down 
a

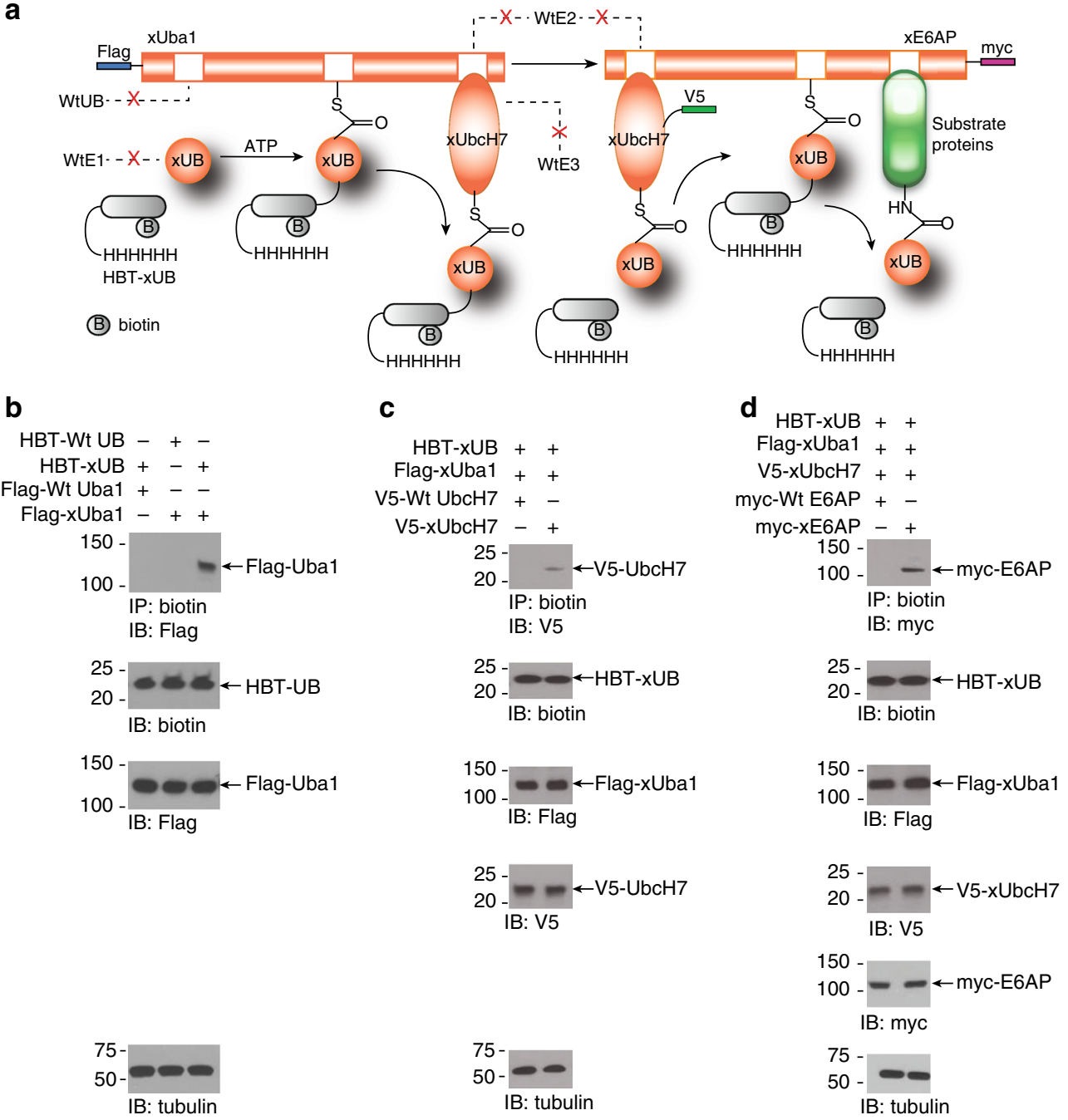

Fig. 4 Orthogonality of the E6AP OUT cascade with wt Uba1-UbcH7-E6AP cascade in HEK293 cells. a Scheme showing exclusive transfer of xUB through the OUT cascade of E6AP. xUba1, xUbcH7 and xE6AP are tagged with Flag, V5 and myc tags, respectively. Dotted lines with a red "X" designate none interactions between OUT components and the native E1, E2, and E3 enzymes. xUB is tagged with biotin and $6 \times$ His tags (HBT-xUB). $\mathbf{b} \times \mathrm{xUB}$ and $\mathrm{xUba1}$ formed $x U B-x U b a 1$ conjugate in HEK293 cells. As a result of conjugate formation, flag-xUba1 could be copurified with HBT-xUB by binding to streptavidin beads. In contrast, flag-wt Uba1 could not be copurified with HBT-xUB, neither could flag-xUba1 be copurified with HBT-wt UB in the cells co-expressing the UB-E1 pairs. This suggests that the crossover $x U B-w t$ Uba1 or wt UB-xUba1 pairs are not active in the cell. $\mathbf{c} x U B$ could be transferred through $x U b a 1-$ $x U b c H 7$ pair to form $x U B-x U b c H 7$ conjugate in the cell. Due to the conjugate formation, $V 5-x U b c H 7$ could be copurified with HBT-xUB from cells expressing HBT-xUB, flag-xUba1 and $V 5-x U b c H 7$. In contrast, the expression of HBT-xUB and the crossover pair of xUba1-wt UbcH7 would not generate $x U B-w t$ UbcH7 conjugate, so V5-wt UbcH7 could not be co-purified with HBT-xUB. $\mathbf{d}$ xUB could be transferred through $x U b a 1-x U b c H 7-x E 6 A P$ cascade to form $x U B-x E 6 A P$ conjugate in the cell. As a result, myc-xE6AP could be co-purified with HBT-xUB in cells expressing HBT-xUB and the $x U b a 1-$

$x U b c H 7-x E 6 A P$ cascade. In contrast, coexpression of HBT-xUB with the crossover cascade of $x U b a 1-x U b c H 7-w t$ E6AP would not generate xUB-wt E6AP conjugate, so myc-wt E6AP could not be co-purified with HBT-xUB

with streptavidin beads showed that $\mathrm{xUba1}$ was co-precipitated with HBT-xUB, but wt Ubal could not be co-precipitated with HBT-xUB, neither xUbal could be co-precipitated with HBT-wt UB (Fig. 4b). This suggests that $x U B$ was exclusively reactive with $\mathrm{xUba} 1$ in the cell, and there is no cross activities between $\mathrm{xUB}$ and wt Uba1, or between wt UB and xUba1. To probe the orthogonality at the E1-E2 interface, we co-expressed HBT-xUB with either wt $\mathrm{UbcH} 7$ or $\mathrm{xUbcH} 7$ in cells stably expressing xUba1. We found V5-tagged $\mathrm{xUbcH7}$ could be purified with the streptavidin beads suggesting the formation of HBT$\mathrm{xUB}-\mathrm{xUbcH7}$ conjugate, yet V5-tagged wt UbcH7 could not be co-purified with HBT-xUB (Fig. 4c). This proves that xUba1$\mathrm{xUbcH7}$ was a functional relay for $\mathrm{xUB}$ in the cell, but $\mathrm{xUba1}-\mathrm{wt}$ $\mathrm{UbcH7}$ pair could not mediate xUB transfer to a wt E2. To verify the orthogonality at the E2-E3 interface, we co-expressed HBT-
xUB with either wt E6AP or xE6AP in HEK293 cells stably expressing the xUba1-xUbcH7 pair, and purified the xUBconjugated proteins by streptavidin beads. Myc-tagged xE6AP was co-purified with $\mathrm{xUB}$ suggesting the formation of xUB-xE6AP conjugate, yet no wt E6AP was conjugated with $x U B$ (Fig. 4d). These results prove that $x U b a 1-x U b c H 7-x E 6 A P$ is an orthogonal cascade for the transfer of $\mathrm{xUB}$, and the crossover of $\mathrm{xUB}$ to wt cascades was eliminated.

Profiling the substrates of E6AP in HEK293 cells by OUT. To express the OUT cascade of E6AP in the cell, we screened cell lines stably expressing $\mathrm{xUba} 1, \mathrm{xUbcH7}$ and $\mathrm{xE6AP}$ by lentiviral infection. Western blots of the cell lysate probed with antibodies against each OUT component suggested their adequate 
expression (Supplementary Fig. 4b). We also generated a stable cell line expressing the $\mathrm{xUba} 1-\mathrm{xUbcH7}$ cascade without $\mathrm{xE6AP}$ as a control for the proteomic screen. To initiate $\mathrm{xUB}$ transfer through the OUT cascade, we transduced the two stable cell lines with lentivirus carrying the vector to express HBT-xUB. We then purified cellular proteins conjugated with HBT-xUB sequentially by Ni-NTA and streptavidin affinity columns under strong denaturing conditions (Supplementary Fig. 4a). We found xUba1, $\mathrm{xUbc1}$, and xE6AP are among the proteins retained by tandem purification suggesting the loading of $\mathrm{HBT}-\mathrm{xUB}$ to the engineered E1, E2, and E3 enzymes of the OUT cascade (Supplementary Fig. 4c). We then digested the proteins on the streptavidin beads by trypsin and analyzed the peptide fragments by LC-MS/MS to identify xUB-conjugated proteins. In parallel, we performed tandem purification and proteomic analysis on control cells expressing the $\mathrm{xUba1}-\mathrm{xUbcH7}$ pair without xE6AP (Supplementary Fig. 4d, e). By comparing the two proteomic profiles, we identified proteins that had ratios of peptide-spectrum match (PSM) 2-fold or higher between cells expressing the full E6AP OUT cascade and the control cells. We carried out affinity purification and proteomic screen three times. We found 130 proteins repeatedly appearing in all three screens with PSM ratio $\geq 2$ (Supplementary Data 1). These proteins are likely the direct ubiquitination targets of E6AP.

Among the E6AP targets identified, we found previously identified substrates such as the UV excision repair protein HHR23A (RAD23A) and HHR23B (RAD23B), proteasomal ubiquitin receptor ADRM1, 26S proteasome non-ATPase regulatory subunit 4 (PSMD4 or Rpn10), 26S proteasome AAA-ATPase subunit Rpt5 (PSMC3), and E3 ligase RING2 (RNF2 or Ring1B) ${ }^{24-27}$. Ingenuity Pathway Analysis (IPA) of the proteins from the OUT screen showed that E6AP targets have a significant association with a variety of canonical pathways (Supplementary Data 2). It is intriguing that several associated pathways mediate cell cycle control and chromosome replication, matching the role of E6AP in viral oncogenesis. IPA also identified 8 protein networks that are significantly associated with E6AP substrates (Supplementary Data 3). The identified networks are related to cell death and survival, DNA replication, recombination, and repair, cellular growth and proliferation, and nervous system development and function.

We used the CRAPome database to evaluate whether proteins non-specifically bound to the affinity resins were among the targets identified by OUT. CRAPome selects non-specific binders in proteomic experiments based on the frequency of their appearance in pull-down experiments with various bait proteins under non-denaturing conditions ${ }^{28}$. In contrast, we used strong denaturing condition to purify $\mathrm{xUB}$-conjugated proteins in the OUT screen. Nevertheless, we found 2 of the 130 E6AP targets identified by OUT have a frequency higher than $34 \%$ in the CRAPome database (Supplementary Data 4). We verified that one of them, PRMT5, is a E6AP target (see below). We also repeatedly identified 35 proteins in control cells without expression of $\mathrm{xE6AP}$, and they were not present among $\mathrm{xUB}$ conjugated proteins purified from cells expressing the full OUT cascade of E6AP (Supplementary Data 5).

In vitro and in vivo validation of the E6AP substrates. We found some key signaling enzymes such as kinases MAPK1, CDK1, CDK4, protein Arg methyltransferase 5 (PRMT5), transcription factor $\beta$-catenin, and FAS-associated factor UbxD8 are likely substrates of E6AP (Supplementary Data 1). We thus assayed if E6AP targets them for ubiquitination and regulates their stabilities in the cell. We first used the wt UB transfer cascade Uba1-UbcH7-E6AP to test if the potential substrate proteins could be modified by wt UB in vitro. We expressed and purified the potential substrates from E. coli cells, and found that they were ubiquitinated by E6AP to different extents: CDK1, CDK4 and $\beta$-catenin were strongly ubiquitinated by E6AP with the formation of high molecular weight bands, while MAPK1, PRMT5, and UbxD8 mainly generated species with one or two conjugated UBs (Fig. 5). As a positive control, E6AP-catalyzed ubiquitination of HHR23A, a previously identified E6AP substrate, was confirmed (Fig. 5g) ${ }^{26}$. Protein expressed in E. coli cells may not bear the proper posttranslational modifications for E6AP recognition, so the in vitro assays may not reflect the real activity of E6AP with the substrate proteins. We thus tested whether the potential substrates are targeted for ubiquitination by E6AP in the cell.

We inhibited E6AP expression in HEK293 cells with lentivirus delivering the anti-E6AP shRNA. We also overexpressed E6AP in blank HEK293 cells and cells harboring the anti-E6AP shRNA. Cells were treated with proteasome inhibitor MG132 before harvesting to inhibit protein degradation. Ubiquitination levels of various substrates in different cell populations were revealed by immunoprecipitation with substrate-specific antibodies and immunoblotting with an anti-UB antibody. Comparing to the parental HEK293 cells, cells expressing anti-E6AP shRNA had significantly lower levels of poly-ubiquitinated forms of MAPK1, CDK1, CDK4, PRMT5, $\beta$-catenin, and UbxD8 (Fig. 6). The polyubiquitination of each target protein in the HEK293 cells harboring the anti-E6AP shRNA can be restored by overexpressing E6AP in the cell. Furthermore, HEK293 cells with over-expression of E6AP gave rise to more intense polyubiquitination of MAPK1, CDK1, CDK4, $\beta$-catenin, and UbxD8 comparing to the parental HEK293 cells. The known E6AP substrate HHR23A showed similar dependence on E6AP for its ubiquitination in the HEK293 cell. These results prove that the potential E6AP substrates identified by the OUT screen are indeed E6AP targets in the cell.

To probe whether E6AP-mediated ubiquitination would signal protein degradation, we transiently transfected HEK293 cells with varying amounts of wt E6AP plasmid. Western blot of the cell lysates with an anti-E6AP antibody showed an increased E6AP expression in the cells receiving more plasmid DNA. In parallel, the levels of CDK1, CDK4, PRMT5, and UbxD8 were significantly reduced in comparison to the control cells without transfection of the E6AP plasmid (Fig. 7a, b). We also measured the half-lives of the target proteins with the cycloheximide (CHX) chase assay. Cells expressing E6AP were treated with $\mathrm{CHX}$ to inhibit protein synthesis and the substrate levels in the cell were measured by immunoblotting with anti-substrate antibodies at different time points. We found that the turnover of PRMT5, CDK1, CDK4, and UbxD8 was significantly faster in cells overexpressing E6AP compared to control cells transfected with the same amount of empty plasmid (Fig. 7c, d). The level of MAPK1 remained stable despite over-expression of E6AP. MAPK1 is among the 400 most expressed proteins in the cell and its half-life is longer than $68 \mathrm{~h}^{29,30}$. This may explain why E6AP expression had little effect on the level of MAPK1 in the cell. On the other hand, inhibiting endogenous E6AP expression in HEK293 cells by shRNA stabilized PRMT5, CDK1, CDK4, $\beta$ catenin, UbxD8 (Fig. 7c, d). The known E6AP substrate HHR23A was also stabilized with the decreased expression of E6AP.

\section{Discussion}

The large number of E3s $(>600)$ encoded in the human genome reflects the key roles they play in cell regulation. On the other hand, their diversity makes it a significant challenge to identify the direct substrates of individual E3s. Current methods screening 
E3 substrates fall into three categories-affinity binding to E3, monitoring changes in protein stability or ubiquitination levels in response to E3 perturbation, or trapping E3 substrates by covalent or noncovalent interactions (Supplementary Fig. 5). Affinitybased approaches such as co-immunoprecipitation, yeast twohybrid system, and protein microarray have been used to screen E3 substrates based on the binding between E3 and substrates (Supplementary Fig. 5a) ${ }^{31-33}$. They are less specific since the $K_{\mathrm{d}}$ 's of the E3-substrate complexes are around hundreds of $\mu \mathrm{M}$, and the complexes are transient ${ }^{34}$. Also, proteins other than substrates can bind to E3s to function as adaptors or regulators. Still, these methods yielded important substrate profiles of HECT E3 E6AP, Ring E3 anaphase-promoting complex (APC), and the Skp1cullin-F-box (SCF) complex ${ }^{31-33}$. A more direct approach to assign E3 substrates is to correlate changes in E3 activity with the changes in stability or ubiquitination level of cellular proteins (Supplementary Fig. 5b). One method known as "global protein stability profiling (GPS)" tracks the stability of thousands of proteins with a fused fluorescence protein tag, and it has been used to screen substrates of SCF E3s ${ }^{35-37}$. The development of anti-diGly antibody allows affinity enrichment of substrate peptides containing the ubiquitination sites, and comparison of protein ubiquitination levels upon perturbation of E3 activity ${ }^{38}$. Using the quantitative diGly proteomics (QdiGly), substrate profiles of cullin-Ring and Parkin E3s were generated ${ }^{39,} 40$. E3s are also converted to substrate traps so the substrate proteins would still be bound to E3 after UB transfer. This would enable the co-purification of the substrate proteins with E3s. To create "UB-activated interaction traps (UBAIT)", UB was fused to HECT and Ring E3s and it can attack the substrates bound to the E3-UB fusion to generate covalent linkages between E3 and the substrate proteins (Supplementary Fig. $5 \mathrm{c}$ ) ${ }^{41}$. Another design is to fuse the F-box proteins with a series of UB associated domain (UBA) and use them as UB ligase traps ${ }^{42,43}$. As F-box proteins recruit substrates to SCF E3s, the UB chain extending from the substrate would bind to the UBA repeats with high affinity. Purification of proteins bound to F-box-UBA fusion would enrich the substrates of the F-box protein (Supplementary Fig. 5d). E2-E3 fusions has also been used to identify E3 substrates. Ubc12, the E2 enzyme mediating Nedd8 transfer, was fused to the substrate binding domain of Ring E3 XIAP. The fusion protein, known as a "Neddylator", allows Nedd8 transfer to XIAP substrates so the ubiquitination targets of XIAP could be identified among Nedd8modified proteins (Supplementary Fig. 5e) ${ }^{44}$. The development of diverse methods to profile E3 substrates enables the interrogation of E3 function from different perspectives. The substrate profiles generated by various methods could corroborate to reveal the functions of E3s.

Here, we developed a method known as "orthogonal ubiquitin transfer (OUT)" to identify the direct substrates of HECT E3 E6AP (Fig. 4a). In OUT, an affinity-tagged UB variant (xUB) is exclusively transferred through an engineered $x E 1-x E 2-x E 3$ cascade to the substrates of a specific E3. By purifying xUB-modified proteins from the cell and identifying them by proteomics, we would be able to identify the direct substrates of a E3. In this study, we used OUT to identify 130 potential E6AP substrates, and among them, we confirmed MAPK1, PMRT5, CDK1, CDK4, $\beta$-catenin, and UbxD8 are ubiquitinated by E6AP in the HEK293 cells. During the revision of this manuscript, $\beta$-catenin was confirmed as an E6AP substrate by another report ${ }^{45}$. A key advantage of OUT is that it assigns E3 substrates by directly following UB transfer from the E3 to its substrate proteins. Methods based on E3 substrate binding, or change of protein stability upon perturbation of E3 activity, use indirect readouts of substrate ubiquitination to assign E3 substrates. The substrate profiles generated by those methods could be distorted by factors

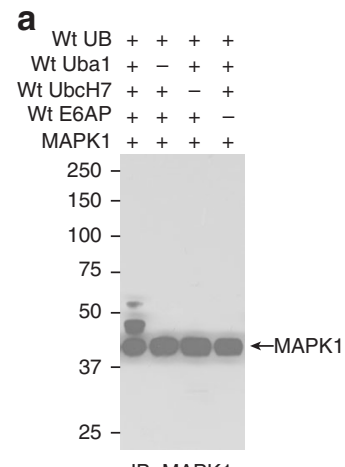

IB: MAPK1

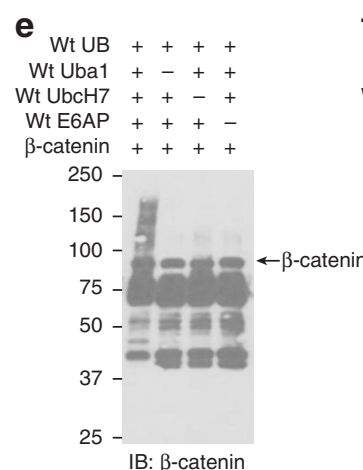

b

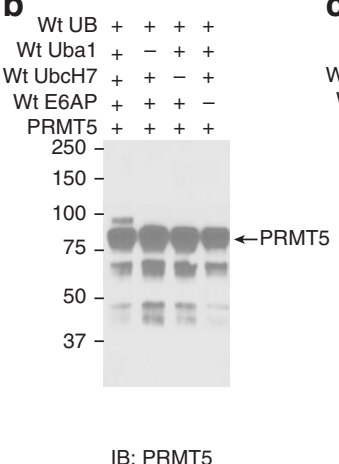

f

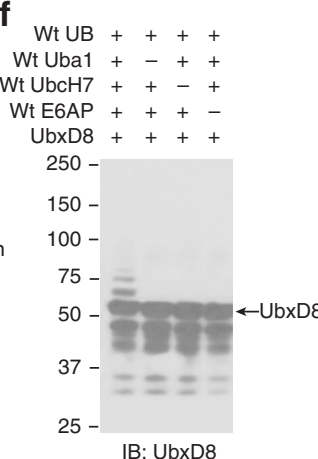

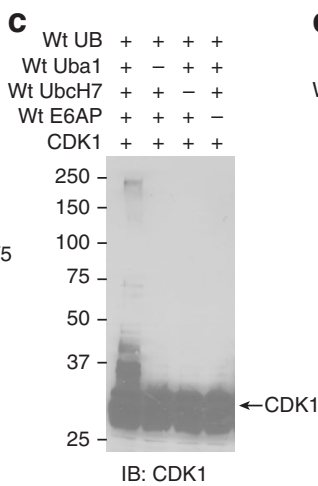

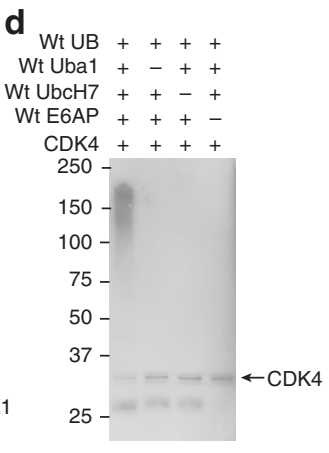

IB: CDK4

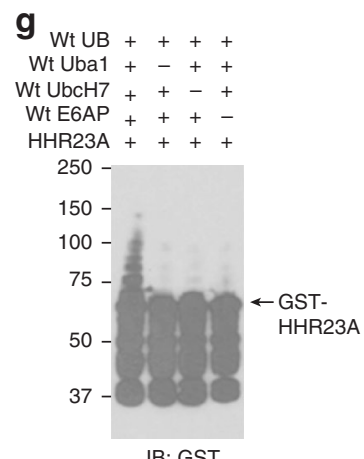

Fig. 5 In vitro assays to test ubiquitination of E6AP substrates identified by OUT. wt UB was transferred through wt Uba1-UbcH7-E6AP cascade to the potential substrate proteins expressed from $E$. coli cells. E6AP ubiquitination of MAPK1 a, PRMT5 b, CDK1 c, CDK4 d, $\beta$-catenin e, and UbxD8 f, were confirmed. E6AP-catalyzed ubiquitination of HHR23A, a previously reported E6AP substrate, was also assayed $\mathbf{g}$. All blots are representative of at least three independent experiments 
a

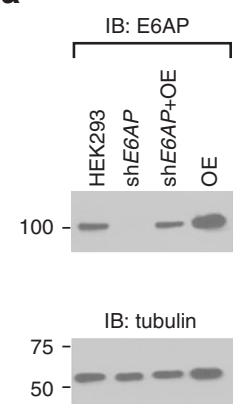

b
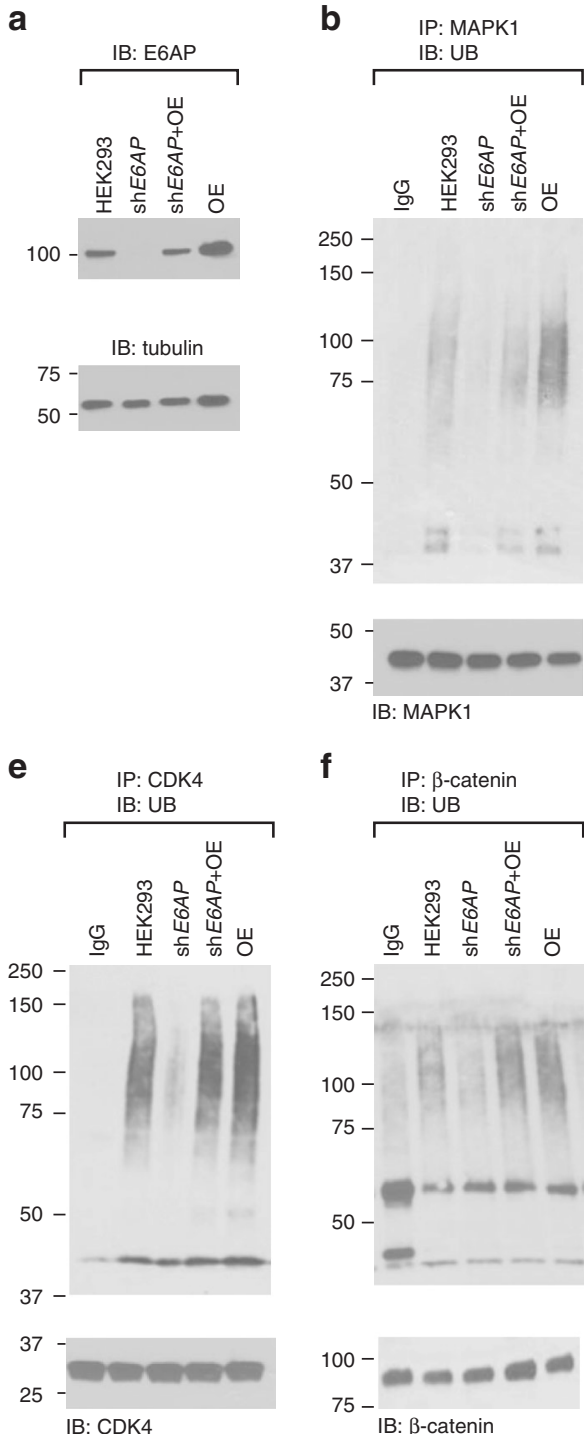

$f$

f

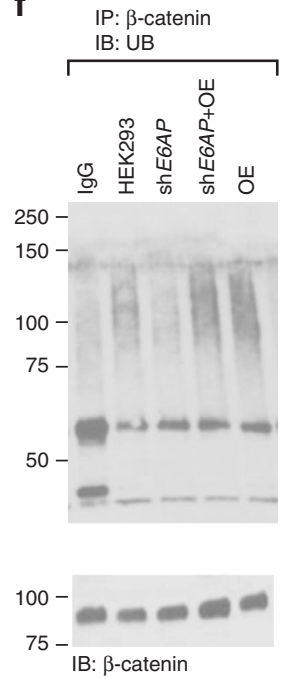

c
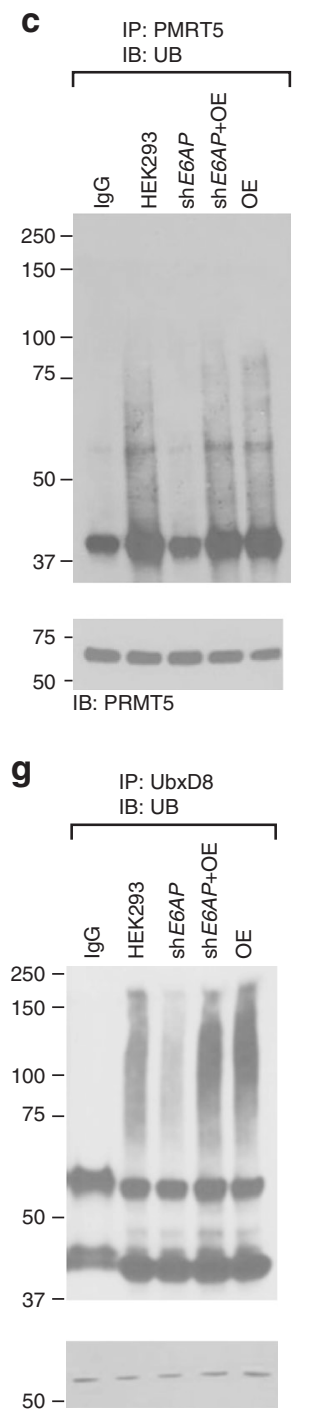

IB: UbxD8 d IP: CDK1

IB: UB
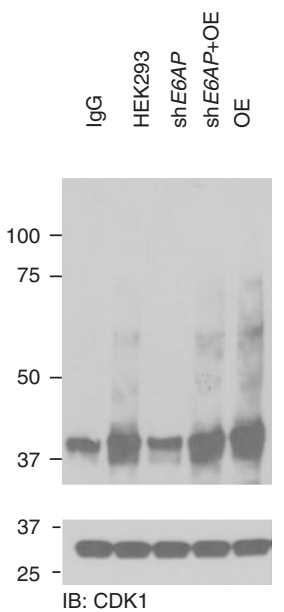

h

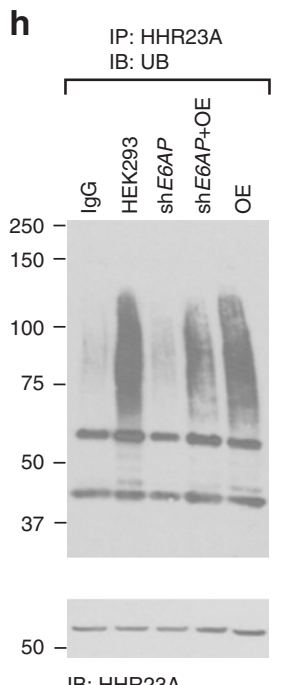

Fig. 6 Cellular assays to test the ubiquitination of E6AP substrates identified by OUT. a Inhibition of E6AP expression in HEK293 cells by shE6AP was confirmed with Western blot probed with an antibody against E6AP. b-g Ubiquitination of MAPK1 b, PRMT5 c, CDK1 d, CDK4 e, $\beta$-catenin $\mathbf{f}$ and UbxD8 $\mathbf{g}$ in HECK293 cells was assayed by immunoprecipitation with antibodies against each substrate proteins and probing the ubiquitination levels of the proteins with an anti-UB antibody on the western blots. After 1.5-h treatment of cells with MG132, ubiquitination of each target protein was compared among the blank HEK293 cell (HEK293), HEK293 expressing shE6AP (shE6AP), HEK293 expressing both shE6AP and recombinant E6AP (shE6AP + OE), and HEK293 expressing recombinant E6AP (OE). h Ubiquitination of HHR23A, a known E6AP substrate, was assayed as a control. Rabbit IgG was used as a control for immunoprecipitation in $\mathbf{b}$ and $\mathbf{e}$. Mouse IgG was used as a control for immunoprecipitation in $\mathbf{c}, \mathbf{d}$ and $\mathbf{f}-\mathbf{h}$. All blots are representative of at least three independent experiments

such as the binding of adaptor or regulatory proteins to $\mathrm{E} 3$, or the attachment of UB chains of non-degradation signals to the substrates. E3 may also regulate the activities of proteasome and other E3s, thus perturbing the activity of one E3 may affect the degradation or ubiquitination levels of the substrates of other $\mathrm{E} 3 \mathrm{~s}^{24,46-48}$. In this study, we used yeast cell surface display to identify mutations at the E2-binding site of the E6AP HECT domain to generate an $\mathrm{xE2}-\mathrm{xE} 3$ pair for the OUT cascade. The mutations at the E2-binding site of E6AP shall have minimal disturbance to its substrate profile. In comparison with various E3 fusions as substrate traps, the engineered xE6AP would be a better reenactor of the wt E3 in transferring xUB to the substrate proteins to enable their identification by OUT.

OUT has a few limitations. First, each E3 would require its own OUT cascade for substrate identification. The xUB-xE1 pair we engineered can be used for the OUT cascade of various E3s. Due to the high sequence homology of the $\mathrm{N}$-terminal helices of the E2s, mutations can be transplanted from $\mathrm{xUbc1}$ to $\mathrm{UbcH} 7$ and UbcH5b to generate $\mathrm{xE1}-\mathrm{xE2}$ pairs ${ }^{17}$. The great diversity of E3s would require the engineering of individual E3s to assemble $\mathrm{xE} 2-\mathrm{xE} 3$ pairs. Here we used yeast cell surface display to identify HECT mutants of E6AP that can pair with xUbcH7. The helical turn we randomized in the E6AP HECT domain is a common element in many HECT enzymes ${ }^{49-52}$. It is possible to generate orthogonal $\mathrm{xE} 2-\mathrm{xE} 3$ pairs by transplanting the mutations from xE6AP to other HECTs E3s. If such strategy is not effective, the yeast selection platform for E6AP HECT could be used to engineer other HECTs such as Smurf1/2, Nedd4-1/2, and Huwe1, all playing important roles in cell regulation ${ }^{5}$. Another limitation of OUT is that co-expression of HBT-xUB and the full $x E 1-x E 2-x E 3$ cascade, although successful in HEK293 cells, maybe a challenge in other cell types. The recently developed 
a

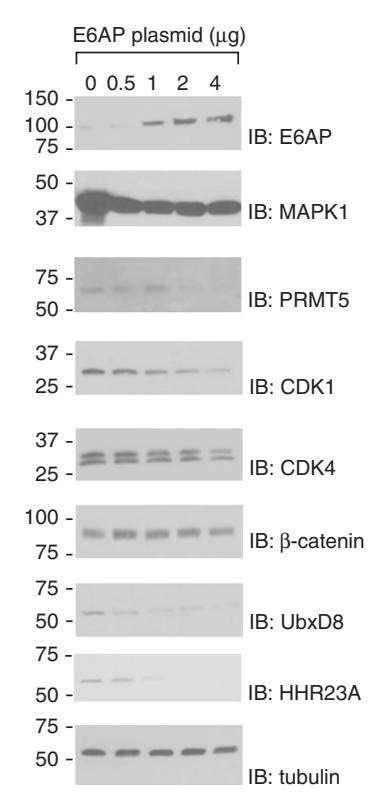

C

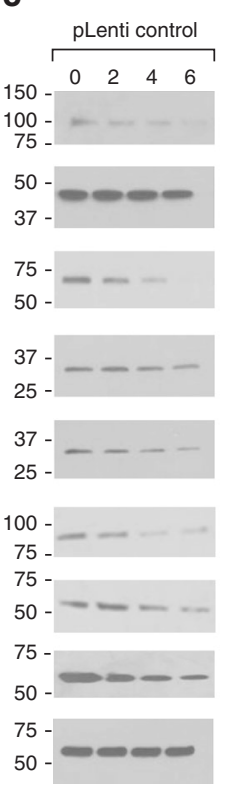

EGAP OE

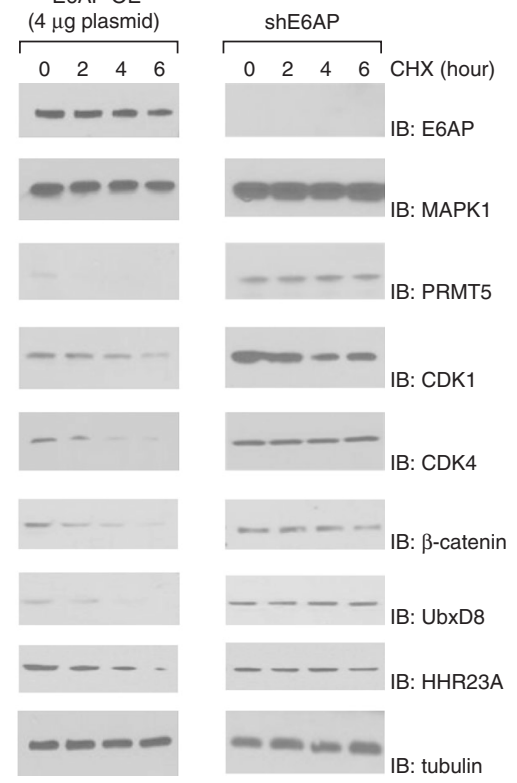

b

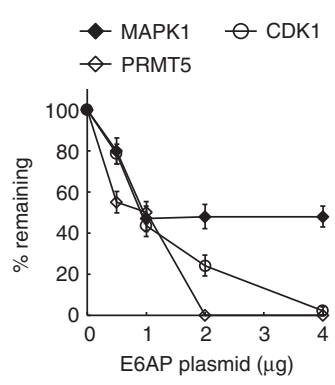

- CDK4 $\triangle$ UbxD8

$-\beta$-catenin $\ominus$ HHR23A

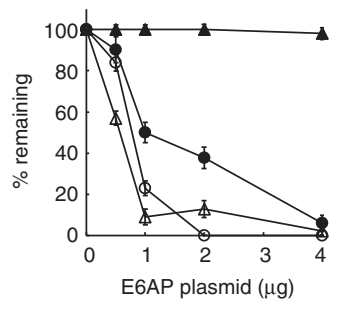

d
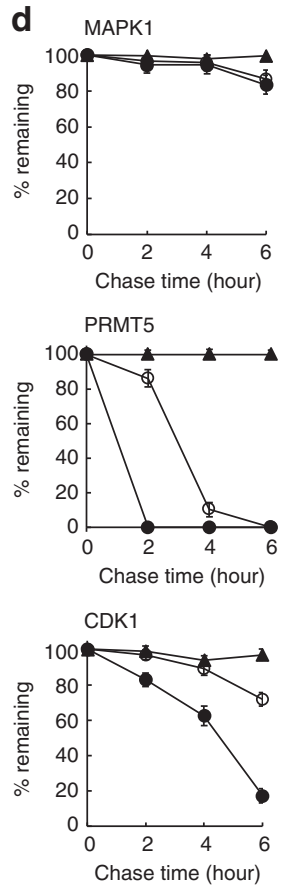
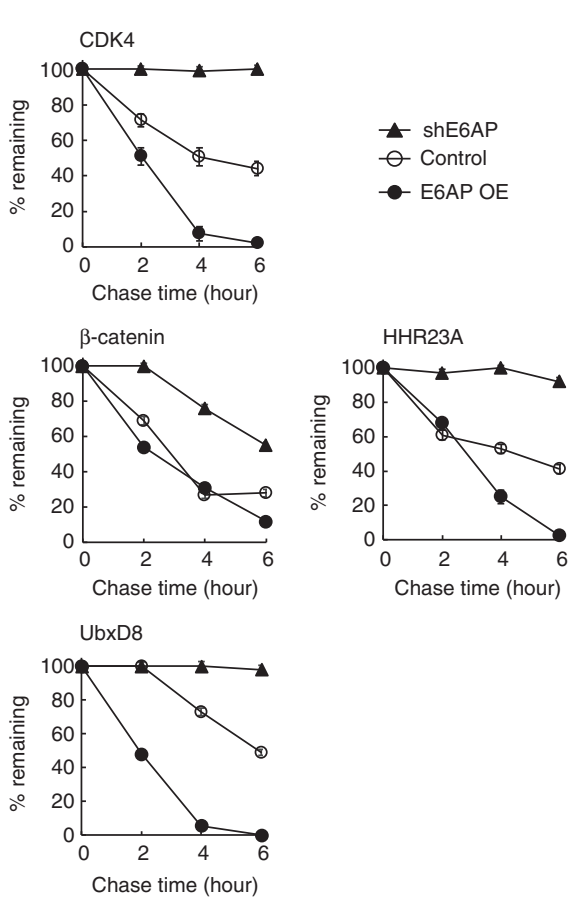

- shE6AP

- Control

- E6AP OE

Fig. 7 Effect of E6AP expression on the stability of the substrate proteins. a E6AP decreases the steady-state levels of the substrate proteins in the HEK293 cells. Cells were transfected with increasing amount of E6AP plasmid. Levels of the E6AP substrates were assayed with immunoblots of the cell lysate probed with substrate-specific antibodies. Approximately $5 \times 10^{6}$ cells were used for each transfection of the E6AP plasmid. b Quantitative analysis of substrate levels in correlation with E6AP expression. Intensity of the bands in a were plotted against the amount of pLenti E6AP plasmid used for transfection assuming $100 \%$ of substrate protein when an empty plasmid was used for mock transfection. Results were the average of three repeats. c E6AP-dependent degradation of substrate proteins assayed by cycloheximide (CHX) chase. HEK293 cells $\left(5 \times 10^{6}\right.$ cells) were transfected with $4 \mu \mathrm{g}$ of pLenti E6AP plasmid with the same amount of empty pLenti plasmid used in the controls. The cells were treated with $100 \mathrm{mg} / \mathrm{ml} \mathrm{CHX} 48 \mathrm{~h}$ after transfection. Cell extracts were collected at $0,2,4$, and $6 \mathrm{~h}$ after incubation with $\mathrm{CHX}$, followed by immunoblotting with substrate-specific antibodies. $\mathbf{d}$ Quantitative analysis of the levels of the substrate proteins in the cell in the CHX chase experiment. Data are representative of three independent experiments. Besides the new substrates identified by OUT, HHR23A, a known E6AP substrate, was assayed for its degradation regulated by E6AP. The vertical bars in $\mathbf{b}$ and $\mathbf{d}$ represent s.e.m. from three independent experiments

genome editing tools such as CRISPR/Cas9 may provide an opportunity to introduce the OUT cascade into the original genetic background to identify E3 substrates ${ }^{53}$.

We found E6AP expression did not affect the stability of MAPK1. The ubiquitination and degradation UbxD8 signaled by
E6AP suggests a role for E6AP in lipid metabolism, since UbxD8, by forming a complex with p97/VCP, regulates lipid droplet size and abundance ${ }^{54}$. Consistently, expression of a dominantnegative E6AP mutant promotes accumulation of lipid droplets ${ }^{55}$, which may involve stabilized UbxD8 due to decreased 
activity of E6AP. The biological significance of E6AP-mediated ubiquitination of CDK1, CDK4 and PRMT5 awaits further investigations. Recent studies have suggested that E6AP is required for cellular senescence, i.e., irreversible exit from the cell cycle, as a physiological response to oxidative stress or oncogene activation $^{56-58}$. Thus, E6AP-mediated ubiquitination of CDKs in the absence of viral oncoproteins may be involved in the senescence response to various cellular stresses. Indeed, Ingenuity Pathway Analysis showed that many of the potential E6AP substrates identified by OUT are associated with pathways and networks relevant to DNA replication, cell cycle control, oncogenic signaling, cell survival/death and development (Supplementary Data 2 and 3). PRMT5 plays a key role in chromatin regulation by methylating Arg residues in histones ${ }^{59}$. Since various studies have indicated the significance of epigenetic changes in cancers and autism spectrum diseases, it would be interesting to determine how E6AP-mediated PRMT5 ubiquitination is involved in the pathobiology of those diseases ${ }^{60-62}$.

\section{Methods}

Reagents. XL1 Blue cells were from Agilent Technologies (Santa Clara, CA, USA). pET-15b and pET-28a plasmids for protein expression were from Novagen (Madison, WI, USA). pCTCON2 plasmid and the yeast strain EBY100 were from K. Dane Wittrup of Massachusetts Institute of Technology ${ }^{21}$. The plasmid with the human Ubal gene was from Wade Harper of Harvard Medical School ${ }^{8}$. The plasmid for E6AP expression was from Jon M. Huibregtse of the University of Texas at Austin ${ }^{63}$. The plasmid for Rsp5 expression was from Linda Hicke of the Northwestern University ${ }^{64}$. pQCXIP HBT-Ubiquitin (26865) was from Addgene (Cambridge, MA, USA) $^{23}$. pLenti-puro plasmid was from Addgene (39478). pLenti4/V5-DEST-zeocin (K498000) and ViraPower Lentiviral Packaging Mix (K4975-00) were from Life Technology. pLenti-puro plasmid was from Addgene (39478). pET-PRMT5 plasmid was provided by Yujun Zheng of University of Georgia, Athens. The mammalian cell expression vectors for MAPK1 (39230), CDK1 (27652), and UbxD8 (53777) and pGEX-HHR23A (10864) were from Addgene. HEK293 cells were from American Tissue Culture Collection (ATCC), and cultured in high-glucose Dulbecco's modified Eagles medium (DMEM) (Life Technologies, Carlsbad, CA, USA, 11965092) with 10\% (v/v) Fetal bovine serum (FBS) (Life Technologies, 11965092). Antibiotics hygromycin, blasticidin, zeocin and puromycin were from GiBCO/Invitrogen (Carlsbad, CA, USA) and RPI (Mount Prospect, IL, USA). Doxycycline was from RPI.

Anti- $\beta$-catenin antibody (sc-65480), anti-CDK1 antibody (sc-54), anti-CDK4 antibody (sc-260), anti-E6AP antibody (sc-25509), anti-HA antibody (sc-7392), anti-HHR23A antibody (sc-365669), anti-MAPK1 antibody (sc-154), anti-Myc antibody (sc-40), anti-p53 antibody (sc-126), anti-PRMT5 antibody (sc-376937), anti- $\alpha$-Tubulin antibody (sc-23948), anti-V5 antibody (sc-271944), anti-UB antibody (sc-8017), anti-UbxD8 antibody (sc-374098) were from Santa Cruz Biotechnology. These antibodies were diluted between 500 and 1,000-fold to probe the Western blots. Goat anti-rabbit IgG-HRP (sc-2004) and goat anti-rabbit IgGHRP (sc-2005) were also from Santa Cruz Biotechnology and were diluted 10,000fold as the secondary antibody for western blotting. Streptavidin-HRP-conjugate was from Life Technologies and was diluted 20,000-fold for western blotting. AntiFlag M2 antibody (F3165) was from Sigma-Aldrich and was diluted 1000-fold for Western blotting. $\mathrm{His}_{6}$-p53 and E6 protein of human papillomavirus type 16 were from Boston Biochem (Cambridge, MA, USA). Oligonucleotides were ordered from Integrated DNA Technologies (Coralville, IA, USA) with their sequences listed in Supplementary Table 1 . Biotin-CoA was prepared by conjugating biotinmaleimide with Coenzyme $\mathrm{A}^{17}$. wt UB and $\mathrm{xUB}$ were expressed as fusions with an $\mathrm{N}$-terminal ybbR tag and were labeled with biotin by the transfer of biotinpantetheinyl group from biotin-CoA to the ybbR tag catalyzed by Sfp phosphopantetheinyl transferase ${ }^{17,65}$.

Construction of the protein expression plasmids. To construct human xUbal mutant with six mutations (Q608R, S621R and D623R, E1037K, D1047K and E1049K), primers Bo184 and Bo185, and Bo 186 and Bo187 were paired to amplify Ubal gene in pET-wt Ubal by polymerase chain reaction (PCR). The amplified PCR fragments had mutations Q608R, S621R and D623R incorporated into the adenylation(A) domain of Ubal. The two PCR fragments were assembled by overlapping PCR and cloned into the $\mathrm{PET}$-wt Ubal vector between restriction sites FseI and EcoRI to generate pET-xUba1(A). To incorporate the three mutations in the UFD domain of Uba1, the mutated Ubal gene in pET-xUbal(A) was PCR amplified with primers Bo13 and Bo73. PCR fragment was digested by restriction enzymes BamHI and EcoRI, and cloned into pET-xUba1(A) to generate pETxUbal with mutations in both the adenylation and the UFD domains. pET-UbcH7 with the R5E and K9E mutations was constructed by PCR amplifying the UbcH7 gene with primers WY9 and WY10 and cloned into pET28a between restriction sites NdeI and XhoI.
HECT domains of Sumrf1, Sumrf2, Nedd4, and E6AP with an N-terminal Flag tag were expressed with the pET28 vector. The genes of the HECT domains were amplified with primers WY1-8 by PCR. The amplified fragments were digested with restriction enzymes SacII and NotI, and cloned into the pET28a plasmid. To express the mutant E6AP HECT from yeast selection, the genes of the mutant HECT domains were PCR amplified with primers WY4 and WY8 from the corresponding pCTCON2 vector, digested by SacII and NotI, and cloned into pET28a. For the expression of full-length E6AP, PCR primers WY21 and WY8 were used to amplify the full-length gene from pGEX4-wt E6AP and cloned into the pET28a-Flag vector between restriction sites SacII and Notl.. For the expression of full-length xE6AP with mutated HECT domains, mutant HECT genes were amplified with primers WY4 and WY8 and cloned into the pET28-E6AP vector between restriction sites PstI and NotI. The CDK1 and CDK4 genes were PCR amplified from their mammalian cell expression plasmids with primer pairs K1-K2 and K3-K4, respectively. The PCR fragments were digested with NedI/XhoI and ScaI/NotI, respectively, and cloned into the pET-28a plasmid. The UbxD8 gene was amplified from a mammalian expression plasmid with primers LZ1 and LZ2, digested with NdeI and NotI, and cloned into pET28. The $\beta$-catenin gene was loned into pGEX plasmid. The pET or PGEX plasmids were transformed into BL21(DE3) pLysS chemical competent cells (Invitrogen) for protein expression.

Construction of the E6AP library. The gene of the E6AP HECT domain was PCR amplified from $\mathrm{pET}$-E6AP with primers WY11 and WY12. The amplified fragment was double-digested with NheI and XhoI, and cloned into pCTCON2 plasmid to generate pCTCON2-E6AP HECT. To generate E6AP library in the pCTCON2 plasmid, the E6AP HECT domain gene was PCR amplified with WY13 and WY14 to incorporate randomized codons at residues $651,652,653,654$, and 656 . The PCR fragment amplified with WY13 and WY14 was combined with fragment amplified with WY11 and WY12 to assemble the HECT library gene by overlapping extension with primers WY11 and WY12. The amplified library gene was digested with NheI and XhoI, and cloned into the pCTCON2 vector. Transformation of the plasmid library into XL1 blue electro-competent cells afforded a library of $2.0 \times 10^{8}$ in diversity, large enough to cover all the mutants with randomized residues replacing D651, D652, M653, M654 and T656 in the E6AP HECT domain. Transformed XL1 blue cells were plated on LB-ampicillin plates (LB agar supplemented with $100 \mu \mathrm{g} \mathrm{mL}^{-1}$ ampicillin) and allowed to grow at $37^{\circ} \mathrm{C}$ overnight. Colonies growing on the plate were scraped and the library DNA was extracted with the Plasmid Maxiprep Kit (Qiagen).

Yeast display of the E6AP library. The E6AP library in pCTCON2 was chemically transformed into EYB100 yeast cells ${ }^{66,67}$. Briefly, yeast cells were first cultured at $30^{\circ} \mathrm{C}$ in $200 \mathrm{ml} \mathrm{YPD}(20 \mathrm{~g}$ dextrose, $20 \mathrm{~g}$ peptone, and $10 \mathrm{~g}$ yeast extract in $1 \mathrm{~L}$ deionized water, sterilized by filtration) to an optical density at $600 \mathrm{~nm}$ (OD600) around 0.5 . The cells were then pelleted at $1,000 \times g$ for $5 \mathrm{~min}$. Cells were washed by $20 \mathrm{~mL}$ TE ( $100 \mathrm{mM}$ Tris base, $10 \mathrm{mM}$ EDTA, pH 8.0 ) and $20 \mathrm{~mL} \mathrm{LiOAc-TE}$ (100 mM LiOAc in TE), before resuspension in approximately $800 \mu \mathrm{L}$ LiOAc-TE. A typical transformation reaction contained a mixture of $1 \mu \mathrm{g}$ pCTCON2 plasmid DNA, $2 \mu \mathrm{L}$ denatured single-stranded carrier DNA from salmon testes (SigmaAldrich), $25 \mu \mathrm{L}$ re-suspended yeast competent cells, and $300 \mu \mathrm{L}$ polyethylene glycol (PEG) solution (40\% (w/v) PEG 3350 in LiOAc-TE). To achieve a library size of $10^{6}, 30$ transformations were set up in parallel. Control was also prepared in which the pCTCON2 plasmid was excluded. Both the transformation reactions and the control were incubated at $30^{\circ} \mathrm{C}$ for $1 \mathrm{~h}$ and then at $42^{\circ} \mathrm{C}$ for $20 \mathrm{~min}$. Cells in each transformation were pelleted by centrifuging at $1000 \times g$ for $30 \mathrm{~s}$ and re-suspended in $20 \mathrm{~mL}$ SDCAA medium $(2 \%(\mathrm{w} / \mathrm{v})$ dextrose, $6.7 \mathrm{~g}$ Difco yeast nitrogen base without amino acids, $5 \mathrm{~g}$ Bacto casamino acids, $50 \mathrm{mM}$ sodium citrate, and $20 \mathrm{mM}$ citric acid monohydrate in $1 \mathrm{~L}$ deionized water, sterilized by filtration). Yeast cells were re-suspended, pooled together into $1 \mathrm{~L}$ SDCAA medium, and allowed to grow at $30^{\circ} \mathrm{C}$ over a 2 -day period to an OD600 above 5 . For long-term storage of the yeast library, $20 \mathrm{ml}$ of the yeast culture was aliquoted in $15 \%$ glycerol stock and stored at $-80^{\circ} \mathrm{C}$. To titer the transformation efficiency, $10 \mu \mathrm{L}$ of the re-suspended yeast transformants was serially diluted in SDCAA medium and plated on Trpplates (20 g agar, $20 \mathrm{~g}$ dextrose, $5 \mathrm{~g}(\mathrm{NH} 4)_{2} \mathrm{SO}_{4}, 1.7 \mathrm{~g}$ Difco yeast nitrogen base without amino acids, $1.3 \mathrm{~g}$ drop-out mix excluding $\operatorname{Trp}$ in $1 \mathrm{~L}$ deionized water, and autoclaved). Yeast cells transformed with pCTCON2 plasmids would appear within 2 days of incubation at $30^{\circ} \mathrm{C}$.

Model selection of yeast cells displaying E6AP HECT domain. Yeast cell EYB100 was transformed with pCTCON2- wt E6AP HECT and streaked on a Trp - plate. After two days of incubation at $30^{\circ} \mathrm{C}$, cells were scraped from the Trp-plate to inoculate in a $5 \mathrm{~mL}$ SDCAA culture that was allowed to shake at $30^{\circ} \mathrm{C}$ to reach an initial OD600 of 0.5 . Cells were centrifuged at $1000 \times g$ for $5 \mathrm{~min}$ and induced for E6AP HECT expression by resuspension in $5 \mathrm{~mL} \mathrm{SGCAA}(2 \%(\mathrm{w} / \mathrm{v})$ galactose 6.7 $\mathrm{g}$ Difco yeast nitrogen base without amino acids, $5 \mathrm{~g}$ Bacto casamino acids, 38 $\mathrm{mM} \mathrm{Na}_{2} \mathrm{HPO}_{4}$ and $62 \mathrm{mM} \mathrm{NaH}_{2} \mathrm{PO}_{4}$, in $1 \mathrm{~L}$ deionized water, sterilized by filtration). The yeast culture was shaken at $20^{\circ} \mathrm{C}$ for $16-24 \mathrm{~h}$. For analysis of E6AP display on the surface of yeast cells, $10^{6}$ cells were re-suspended in $0.1 \mathrm{~mL}$ Trisbuffered saline (TBS) (25 mM Tris, $\mathrm{pH} 7.5,150 \mathrm{mM} \mathrm{NaCl}$ ) with $0.1 \%$ bovine serum albumin (BSA). The cells were first labeled with biotin-wt UB based on UB loading on the E6AP HECT domain displayed on the cell surface. $100 \mu \mathrm{L}$ labeling reaction 
was set up with $0.5 \mu \mathrm{M}$ wt Uba1, $5 \mu \mathrm{M}$ wt Ubch7, $0.1 \mu \mathrm{M}$ biotin-wt UB in a buffer containing $10 \mathrm{mM} \mathrm{MgCl}$ and $50 \mathrm{mM}$ Tris- $\mathrm{HCl}(\mathrm{pH} 7.5)$. The reaction was incubated for $2 \mathrm{~h}$ at $30^{\circ} \mathrm{C}$, and then mixed with $100 \mu \mathrm{L} 3 \%$ BSA. A mouse anti-HA antibody (Santa Cruz Biotechnology, sc-7392) was added to the reaction mixture to detect the expression of E6AP tag by binding to the HA tag fused to the Nterminus of the HECT domain. The anti-HA antibody was added to a final concentration of $10 \mu \mathrm{g} \mathrm{mL}^{-1}$ and the cells were incubated for overnight at $4{ }^{\circ} \mathrm{C}$. The cells were then washed twice with $0.1 \%$ BSA in TBS and stained with $5 \mu \mathrm{g} \mathrm{mL}^{-1}$ goat anti-mouse antibody conjugated with Alexa Fluor 647 (Life Technologies, A21235) in $0.1 \mathrm{~mL} 0.1 \%$ BSA in TBS. $5 \mu \mathrm{g} \mathrm{mL}^{-1}$ streptavidin conjugated with PE (Life Technologies, S866) was also added to bind to biotin-UB conjugated to the HECT domain. The cell suspension was shielded from light and incubated at $4{ }^{\circ} \mathrm{C}$ for $1 \mathrm{~h}$. After washing twice with $0.1 \%$ BSA in TBS, cells were analyzed on a flow cytometer (BD LSRFortessa) to count the number of cells that were labeled with fluorophore. Cells were also analyzed from control labeling reactions in which the primary anti-HA antibody was excluded from the labeling reaction, or Ubal, $\mathrm{UbcH7}$ or biotin- wt UB was excluded from the UB loading reaction.

Selection of the E6AP library displayed on the yeast cell. The first round of selection of the yeast library was carried out with magnetic-activated cell sorting (MACS). For subsequent rounds of selection, fluorescence-activated cell sorting (FACS) was used. For MACS, $500 \mu \mathrm{L}$ reactions in TBS buffer $(10 \mathrm{mM} \mathrm{MgCl}, 50$ $\mathrm{mM}$ Tris- $\mathrm{HCl}, \mathrm{pH} 7.5$ ) with $0.1 \%$ BSA were set up with approximately $5 \times 10^{7}$ yeast cells displaying the E6AP library. The reaction mixture contains $5 \mu \mathrm{M}$ xUba1, 20 $\mu \mathrm{M} x \mathrm{xUcH} 7$ and $5 \mu \mathrm{M}$ biotin-xUB to enable $\mathrm{xUB}$ transfer to HECT. After reacting for $2 \mathrm{~h}$ at $30^{\circ} \mathrm{C}$, cells were pelleted by centrifugation and re-suspended in fresh $0.1 \%$ BSA in TBS. This procedure was repeated twice to remove biotin-xUB that was not covalently conjugated to yeast cells. After washing, cells were mixed with $100 \mu \mathrm{L}$ streptavidin-coated microbeads provided by the mMACS Streptavidin Starting Kit (Miltenyi Biotec, 130-091-287) in a total volume of $1 \mathrm{~mL}$ TBS with $0.1 \%$ BSA. Cell suspension was shielded from light and incubated at $4{ }^{\circ} \mathrm{C}$ for $1 \mathrm{~h}$. The suspension of the cells and magnetic beads were then added to $30 \mathrm{~mL}$ of $0.1 \%$ BSA in TBS. The cell suspension was pelleted by centrifugation at $500 \times \mathrm{g}$ for 10 min. The supernatant was aspirated, and the cell pellet including the magnetic beads was re-suspended in $500 \mu \mathrm{L} 0.1 \%$ BSA-TBS. Yeast cells bound to magnetic beads by biotin-streptavidin interaction were captured by a magnet according to manufacturer's instructions, and the beads were washed with $0.1 \%$ BSA in TBS. Cells bound to the magnetic beads were eluted into $5 \mathrm{ml}$ SDCAA medium supplemented with $100 \mu \mathrm{g} \mathrm{mL}^{-1}$ ampicillin, and $50 \mu \mathrm{g} / \mathrm{mL}$ kanamycin, and were cultured at $30^{\circ} \mathrm{C}$ overnight. In parallel, library cells were bound to primary and secondary antibodies to evaluate the display of HECT mutants on the yeast cell surface.

For the 2nd round of selection, the library cells amplified from the first round were incubated with $1 \mu \mathrm{M}$ xUba1, $10 \mu \mathrm{M}$ xUbcH7, and $5 \mu \mathrm{M}$ biotin-xUB for $1 \mathrm{~h}$. After loading biotin-xUB to the HECT domain, the cells were labeled with $10 \mu \mathrm{g}$ $\mathrm{mL}^{-1}$ mouse anti-HA antibody for $1 \mathrm{~h}$. Next, the cells were washed three times, each time with $1 \mathrm{~mL} 0.1 \%$ BSA in TBS. The cells were incubated with $5 \mu \mathrm{g} \mathrm{mL}^{-1}$ goat anti-mouse antibody conjugated with Alexa Fluor 647 and $5 \mu \mathrm{g} \mathrm{mL}^{-1}$ streptavidin conjugated with PE as secondary reagents. After incubation for $1 \mathrm{~h}$ at $4{ }^{\circ} \mathrm{C}$, the cells were pelleted, washed twice each time with $1 \mathrm{~mL} 0.1 \%$ BSA in TBS. Cells doubly labeled with both PE and Alexa Fluor 647 fluorophores were collected by FACS (BD FACSAria llu). The cells collected were pelleted and re-suspended in SDCAA supplemented with $100 \mu \mathrm{g} \mathrm{mL}^{-1}$ ampicillin and $50 \mu \mathrm{g} \mathrm{mL}^{-1}$ kanamycin. The cells were allowed to grow at $30^{\circ} \mathrm{C}$ to an $\mathrm{OD}_{600}$ between 1 and 2 . Glycerol stock of the cells were prepared and they were used to inoculate yeast cell culture for the next round of selection.

In subsequent rounds of selection, the concentration of $\mathrm{xUba} 1, \mathrm{xUbcH} 7$ and biotin-UB in the reaction were decreased in each round. For the 6th rounds of selection, $0.5 \mu \mathrm{M}$ xUba1, $5 \mu \mathrm{M} \times \mathrm{xUbH} 7$ and $0.1 \mu \mathrm{M} \times \mathrm{xUB}$ was used. The gate for sorting the yeast cells also became more stringent in each round with the sixth round only collecting the top $0.5 \%$ of doubly labeled cells. After six rounds of cell selection, the collected cells were grown in an SDCCA medium to an $\mathrm{OD}_{600}$ around 0.5. Zymoprep II Yeast Plasmid Miniprep Kit (Zymo Research, D2004) was used to extract the pCTCON2 plasmid DNA. The plasmid was transformed into XL1 blue competent cells. Plasmid DNA from individual colonies were miniprepped, and sequenced to reveal the mutations in the selected HECT domain clones.

Construction of lentiviral vector and stable cell lines. To generate pLenti6-V5D-TOPO-Asc1-hygromycin-HBT-(x)UB plasmids, HBT tag was sub-cloned from pQCXIP HBT-UB and fused with DNA fragments of human wt UB or xUB by PCR. The assembled DNA fragment was cloned into the pLenti6 plasmid with a hygromycin resistant gene. Genes of xUba1, xUbcH7 and xE6AP were cloned into lentiviral vectors for the selection of stable cell lines. Flag-xUbal gene was PCR amplified with primer WY15 and primer WY16 and cloned into pLenti6-V5-DTOPO-Flag-Ascl-blasticdin vector between restriction sites EcoRI and AscI. V5 xUbcH7 gene was PCR amplified from $\mathrm{pET}$-xUbcH7 with PCR primers WY17 and WY18, digested with restriction enzymes Afe1 and NheI, and cloned into pLenti4V5-D backbone with a zeocin-resistance gene. The gene of xE6AP was PCR amplified with primers WY19 and WY20 and cloned into a pLenti-puromycin vector with a myc tag between restriction sites NheI and XhoI. Virus packaging, virus infection and selection of stable cell lines were performed according to the manufacturer's protocol for the ViraPower Lentiviral Expression System. Stable HEK293 cell lines expressing Flag-xUba1 and V5-xUbcH7 were selected with 10 $\mu \mathrm{g} \mathrm{mL}{ }^{-1}$ blasticidin and $100 \mu \mathrm{g} \mathrm{mL}^{-1}$ Zeocin, respectively. Stable cell line for MycxE6AP was selected with $1 \mu \mathrm{g} \mathrm{mL}{ }^{-1}$ puromycin. Expression of transfected genes was induced by the addition of $1 \mu \mathrm{g} / \mathrm{mL}$ doxycycline to the medium.

Tandem affinity purification of xUB-conjugated proteins. Tandem purification of cellular proteins conjugated to HBT-xUB was performed as following ${ }^{23} .30$ dishes $(10 \mathrm{~cm}$ in diameter) of HEK293 cells stably expressing the xUbal-xUbch7xE6AP cascade were acutely infected with lentivirus HBT-xUB for $72 \mathrm{~h}$. To inhibit proteasome activity, cells were treated with $10 \mu \mathrm{M} \mathrm{MG132}$ for $4 \mathrm{~h}$ at $37^{\circ} \mathrm{C}$. Cells were then washed twice with ice-cold $1 \times \mathrm{PBS}$, pH 7.4, and harvested by cell scraper with buffer A ( $8 \mathrm{M}$ urea, $300 \mathrm{mM} \mathrm{NaCl}, 50 \mathrm{mM}$ Tris, $50 \mathrm{mM} \mathrm{NaH} \mathrm{PO}_{4}, 0.5 \% \mathrm{NP}$ 40, $1 \mathrm{mM}$ PMSF and $125 \mathrm{U} / \mathrm{ml}$ Benzonase, $\mathrm{pH}$ 8.0). For Ni-NTA purification, cell lysates were centrifuged at $15,000 \times g$ for $30 \mathrm{~min}$ at room temperature. $35 \mu \mathrm{L}$ of $\mathrm{Ni}^{2}$ ${ }^{+}$Sepharose beads (GE Healthcare) for each $1 \mathrm{mg}$ of protein lysates were added to the clarified supernatant. After incubation overnight at room temperature in buffer A with $10 \mathrm{mM}$ imidazole on a rocking platform, $\mathrm{Ni}^{2+}$ Sepharose beads were pelleted by centrifugation at $100 \times g$ for $3 \mathrm{~min}$ and washed sequentially with 20 -bead volume of buffer A ( $\mathrm{pH} \mathrm{8.0),} \mathrm{buffer} \mathrm{A} \mathrm{(} \mathrm{pH} 6.3$ ), and buffer A (pH 6.3) with $10 \mathrm{mM}$ imidazole. After washing the beads, proteins were eluted twice with 5-bead volume of buffer B ( $8 \mathrm{M}$ Urea, $200 \mathrm{mM} \mathrm{NaCl}, 50 \mathrm{mM} \mathrm{Na}_{2} \mathrm{HPO}_{4}$, 2\% SDS, $10 \mathrm{mM}$ EDTA, $100 \mathrm{mM}$ Tris, $250 \mathrm{mM}$ imidazole, $\mathrm{pH}$ 4.3). For streptavidin purification, the $\mathrm{pH}$ of the eluted fractions were adjusted to $\mathrm{pH}$ 8.0. $50 \mu \mathrm{L}$ streptavidin-sepharose beads (Thermo Scientific, Rockford, IL) was added to the elution to bind ubiquitinated proteins. After incubation on a rocking platform overnight at room temperature, streptavidin beads were pelleted and washed sequentially with $1.5 \mathrm{~mL}$ buffer C ( $8 \mathrm{M}$ Urea, $200 \mathrm{mM} \mathrm{NaCl}, 2 \%$ SDS, $100 \mathrm{mM}$ Tris, pH 8.0), buffer D (8 M Urea, $1.2 \mathrm{M}$ $\mathrm{NaCl}, 0.2 \%$ SDS, $100 \mathrm{mM}$ Tris, $10 \% \mathrm{EtOH}, 10 \%$ Isopropanol, $\mathrm{pH} 8.0$ ) and buffer $\mathrm{E}$ (8 $\mathrm{M}$ urea, $100 \mathrm{NH}_{4} \mathrm{HCO}_{3}, \mathrm{pH}$ ).

Sample digestion. Residual buffer E was removed and $200 \mu \mathrm{L}$ of $50 \mathrm{mM}$ $\mathrm{NH}_{4} \mathrm{HCO}_{3}$ was added to each sample, which were then reduced with dithiothreitol (final concentration $1 \mathrm{mM}$ ) for $30 \mathrm{~min}$ at $25^{\circ} \mathrm{C}$. This was followed by $30 \mathrm{~min}$ of alkylation with $5 \mathrm{mM}$ iodoacetamide in the dark. The samples were then digested with $1 \mu \mathrm{g}$ of lysyl endopeptidase (Wako) at room temperature for $2 \mathrm{~h}$ and further digested overnight with 1:50 (w/w) trypsin (Promega) at room temperature. Resulting peptides were acidified with $25 \mu \mathrm{L}$ of $10 \%$ (v/v) formic acid (FA) and $1 \%$ (v/v) triflouroacetic acid (TFA) and desalted with a Sep-Pak C18 column (Waters). Briefly, the Sep-Pak column was washed with $1 \mathrm{~mL}$ of methanol and $1 \mathrm{~mL}$ of $50 \%$ $(\mathrm{v} / \mathrm{v})$ acetonitrile $(\mathrm{ACN})$. Equilibration was performed with 2 rounds of $1 \mathrm{~mL}$ of $0.1 \%(\mathrm{v} / \mathrm{v})$ TFA in water. The acidified peptides were then loaded and the column washed with 2 rounds of $1 \mathrm{~mL} 0.1 \%(\mathrm{v} / \mathrm{v})$ TFA. Elution was carried out by 2 rounds of $50 \%(\mathrm{v} / \mathrm{v}) \mathrm{ACN}(400 \mu \mathrm{L}$ each) and the resulting peptide eluent dried under vacuum.

LC-MS/MS analysis. Liquid chromatography coupled to tandem mass spectrometry (LC-MS/MS) on an Orbitrap Fusion mass spectrometer (ThermoFisher Scientific, San Jose, CA) was performed at the Emory Integrated Proteomics Core (EIPC) ${ }^{68,69}$. The dried samples were re-suspended in $10 \mu \mathrm{L}$ of loading buffer $(0.1 \%$ $(\mathrm{v} / \mathrm{v})$ formic acid, $0.03 \%(\mathrm{v} / \mathrm{v})$ trifluoroacetic acid, $1 \%(\mathrm{v} / \mathrm{v})$ acetonitrile), vortexed for $5 \mathrm{~min}$ and centrifuged down at maximum speed for $2 \mathrm{~min}$. Peptide mixtures (2 $\mu \mathrm{L}$ ) were loaded onto a $25 \mathrm{~cm} \times 75 \mu \mathrm{m}$ internal diameter fused silica column (New Objective, Woburn, MA) self-packed with $1.9 \mu \mathrm{m}$ C18 resin (Dr. Maisch, Germany). Separation was carried out over a 2-hour gradient by a Dionex Ultimate 3000 RSLCnano system at a flowrate of $350 \mathrm{~nL} / \mathrm{min}$. The gradient ranged from 3 to $80 \%(\mathrm{v} / \mathrm{v})$ buffer B (buffer A: $0.1 \%(\mathrm{v} / \mathrm{v})$ formic acid in water, buffer B: $0.1 \%(\mathrm{v} / \mathrm{v})$ formic acid in ACN). In each cycle, the mass spectrometer performed a full MS scan followed by as many tandem MS/MS scans allowed within the 3-second time window (top speed mode). Full MS scans were collected in profile mode at 120,000 resolution at $\mathrm{m} / \mathrm{z} 200$ with an automatic gain control (AGC) of 200,000 and a maximum ion injection time of $50 \mathrm{~ms}$. The full mass range was set from 400-1600 $\mathrm{m} / \mathrm{z}$. Tandem MS/MS scans were collected in the ion trap after higher-energy collisional dissociation (HCD) in the ion routing multipole. The precursor ions were isolated with a $0.7 \mathrm{~m} / \mathrm{z}$ window and fragmented with $30 \%$ collision energy. The product ions were collected with the AGC set for 10,000 and the maximum injection time set to $35 \mathrm{~ms}$. Previously sequenced precursor ions within \pm 10 p.p.m. were excluded from sequencing for $20 \mathrm{~s}$ using the dynamic exclusion parameters and only precursors with charge states between $2+$ and $6+$ were allowed.

Database search. All raw data files were processed using the Proteome Discoverer 2.0 data analysis suite (Thermo Scientific, San Jose, CA). The database was downloaded from Uniprot and consists of 90,300 human target sequences. Peptide matches were restricted to fully tryptic cleavage and precursor mass tolerances of \pm 20 p.p.m. Dynamic modifications were set for methionine oxidation $(+15.99492$ $\mathrm{Da})$, asaparagine and glutamine deamidation $(+0.98402 \mathrm{Da})$, lysine ubiquitination $(+114.04293 \mathrm{Da})$ and protein $\mathrm{N}$-terminal acetylation $(+42.03670)$. A maximum of 3 dynamic modifications were allowed per peptide and a static modification of 
+57.021465 Da was set for carbamidomethyl cysteine. The Percolator node within Proteome Discoverer was used to filter the peptide spectral match (PSM) false discovery rate to $1 \%{ }^{70}$.

Bioinformatics analysis. Ingenuity Pathway Analysis (IPA) software (http:/www. ingenuity.com) was used to map and identify the biological networks and molecular pathways with a significant proportion of genes having E6AP ubiquitination targets. Fisher exact test in Ingenuity Pathway Analysis software was used to calculate $p$-values for pathways and networks. The level of statistical significance was set at a $p$-value $<0.05$. IPA was also used to visualize the identified biological networks. Proteins identified by the OUT screen were also analyzed by the CRAPome database (http://www.crapome.org/2 ${ }^{28}$. CRAPome is based on data from interactome studies that carried out affinity purification under denaturing conditions. In contrast, the tandem purification for OUT screens was performed under more stringent denaturing conditions.

Lentiviral silencing of E6AP. Lentiviral GPIZ plasmids encoding shRNAs against E6AP (6 different shRNAs) were obtained from GE Dharmacon (Lafayette, CO), and lentiviruses were produced using the manufacturer's lentivurus packaging system and 293FT cells. HEK293 cells were infected with each lentivirus, followed by selection with puromycin for stable cell populations. The efficiency of gene silencing in each shRNA group was determined by immunoblotting using stable cell populations. For functional restoration, HEK293 cell population stably expressing anti-E6AP shRNA \#1 was infected with the lentivirus packaged with pLenti6-Myc-wt E6AP.

In vitro assay to confirm the substrates of E6AP. All assays were set up in $30 \mu \mathrm{L}$ TBS supplemented with $10 \mathrm{mM} \mathrm{MgCl}_{2}$ and $1.5 \mathrm{mM}$ ATP. In each UB transfer reaction, $5 \mu \mathrm{M}$ of potential substrates (MAPK1, CDK1, CDK4, PRMT5, $\beta$-catenin, and UbxD8) were incubated with $1 \mu \mathrm{M}$ wt Uba1, $5 \mu \mathrm{M}$ wt UbcH7, $10 \mu \mathrm{M}$ E6AP, and $20 \mu \mathrm{M}$ wt UB for $2 \mathrm{~h}$ at $30^{\circ} \mathrm{C}$. The reactions were quenched by boiling in Laemmli buffer with BME, and analyzed by Western blotting probed with substrate-specific antibodies.

Co-immunoprecipitation and to confirm E6AP substrates. Transfection of pLenti-E6AP into the HEK293 cells was conducted with the Lipofectamine ${ }^{\circledR} 2000$ according to the manufacturer's protocol. To immunoprecipitate substrate proteins, cells were treated with $10 \mu \mathrm{M}$ MG132 (American Peptide, Sunnyvale, CA) for $90 \mathrm{~min}$ at 72 -h post-transfection. HEK293 cells (80-90\% confluent monolayer in $75 \mathrm{~cm}^{2}$ cell culture flask) expressing control plasmid, shE6AP, shE6AP + E6AP cDNA, and E6AP cDNA were washed twice with ice-cold PBS, pH 7.4. $1 \mathrm{~mL}$ icecold RIPA buffer was added to cell monolayer and incubated with cell for at $4{ }^{\circ} \mathrm{C}$ for $10 \mathrm{~min}$. The cells were disrupted by repeated aspiration through a 21 -gauge needle. The cell lysate was transferred to a $1.5 \mathrm{~mL}$ tube. The cell debris was pelleted by centrifugation at 13,000 r.p.m. for $20 \mathrm{~min}$ at $4{ }^{\circ} \mathrm{C}$ and the supernatant was transferred to a $1.5 \mathrm{~mL}$ centrifuge tube and precleared by adding $1.0 \mu \mathrm{g}$ of the appropriate control IgG (normal mouse or rabbit IgG corresponding to the hos species of the primary antibody). $20 \mu \mathrm{L}$ of re-suspended volume of Protein $\mathrm{A} / \mathrm{G}$ PLUS-agarose was added to the supernatant and incubation was continued for 30 $\min$ at $4^{\circ} \mathrm{C}$. The agarose beads were pelleted by centrifugation at $350 \times g$ for $5 \mathrm{~min}$ at $4{ }^{\circ} \mathrm{C}$. From the cleared cell lysate, volume containing $2 \mathrm{mg}$ total protein was transferred to a new tube. $30 \mu \mathrm{L}$ (i.e., $6 \mu \mathrm{g}$ ) primary antibody was then added and incubation was continued for $1 \mathrm{~h}$ at $4^{\circ} \mathrm{C}$. After incubation, $50 \mu \mathrm{L}$ of re-suspended volume of Protein A/G PLUS-Agarose was added. The tubes were capped and incubate at $4{ }^{\circ} \mathrm{C}$ on a rocking platform overnight. The agarose beads were pelleted by centrifugation at $350 \times g$ for $5 \mathrm{~min}$ at $4{ }^{\circ} \mathrm{C}$. The beads were then washed 4 times each time with $1.0 \mathrm{~mL}$ PBS. After the final wash, the beads were re-suspended in 40 $\mu \mathrm{L} 1 \times$ Laemmli buffer with BME. The samples were boiled for $5 \mathrm{~min}$ and analyzed by SDS-PAGE and Western blot probed with antibodies specific for the substrate proteins.

E6AP induced protein degradation. To examine the effects of E6AP on steadystate levels of the substrates, HEK293 cells $\left(5 \times 10^{6}\right.$ cells $)$ were transiently transfected with $0.5,1,2$, and $4 \mu \mathrm{g}$ pLenti-wt E6AP with Lipofactamine 2000. Cells were harvested at 48-hour post-transfection and the amount of substrate proteins in the cell lysate was assayed by immunoblotting with substrate-specific antibodies. For cycloheximide $(\mathrm{CHX})$ chase assays, HEK293 cells $\left(5 \times 10^{6}\right.$ cells $)$ were transiently transfected with $4 \mu \mathrm{g}$ empty pLenti or pLenti-E6AP plasmids. After $48 \mathrm{~h}$, cells were treated with $100 \mu \mathrm{g} / \mathrm{mL}$ CHX to block de novo protein synthesis and the cells were harvested after variable length of incubation time with CHX. The amount of substrate proteins in the cell were assayed by immunoblotting with antibodies against each substrate proteins. Protein levels were normalized to tubulin. Alternatively, CHX chase assays were performed on HEK293 cells stably expressing anti-E6AP shRNA to measure the effect of decreased expression of E6AP on substrate stability. Uncropped scans of the Western blots are presented in Supplementary Figs. 6-11.
Data availability. The proteomics data supporting the findings of this study have been deposited to the ProteomeXchange Consortium via the PRIDE partner repository with the identifier PXD005584 (http://proteomecentral. proteomexchange.org). All other data are available from the corresponding authors upon reasonable request.

Received: 16 December 2016 Accepted: 27 October 2017

Published online: 20 December 2017

\section{References}

1. Hershko, A. \& Ciechanover, A. The ubiquitin system. Annu. Rev. Biochem. 67, 425-479 (1998).

2. Schulman, B. A. \& Harper, J. W. Ubiquitin-like protein activation by E1 enzymes: the apex for downstream signalling pathways. Nat. Rev. Mol. Cell Biol. 10, 319-331 (2009).

3. Wenzel, D. M., Stoll, K. E. \& Klevit, R. E. E2s: structurally economical and functionally replete. Biochem. J. 433, 31-42 (2011).

4. Hatakeyama, S. \& Nakayama, K. I. U-box proteins as a new family of ubiquitin ligases. Biochem. Biophys. Res. Commun. 302, 635-645 (2003).

5. Rotin, D. \& Kumar, S. Physiological functions of the HECT family of ubiquitin ligases. Nat. Rev. Mol. Cell Biol. 10, 398-409 (2009).

6. Wenzel, D. M., Lissounov, A., Brzovic, P. S. \& Klevit, R. E. UBCH7 reactivity profile reveals parkin and HHARI to be RING/HECT hybrids. Nature 474, 105-108 (2011).

7. Deshaies, R. J. \& Joazeiro, C. A. RING domain E3 ubiquitin ligases. Annu. Rev. Biochem. 78, 399-434 (2009).

8. Jin, J., Li, X., Gygi, S. P. \& Harper, J. W. Dual E1 activation systems for ubiquitin differentially regulate E2 enzyme charging. Nature 447, 1135-1138 (2007).

9. Frescas, D. \& Pagano, M. Deregulated proteolysis by the F-box proteins SKP2 and beta-TrCP: tipping the scales of cancer. Nat. Rev. Cancer 8, 438-449 (2008).

10. Popovic, D., Vucic, D. \& Dikic, I. Ubiquitination in disease pathogenesis and treatment. Nat. Med. 20, 1242-1253 (2014).

11. Scheffner, M., Huibregtse, J. M., Vierstra, R. D. \& Howley, P. M. The HPV-16 E6 and E6-AP complex functions as a ubiquitin-protein ligase in the ubiquitination of p53. Cell 75, 495-505 (1993).

12. Glessner, J. T. et al. Autism genome-wide copy number variation reveals ubiquitin and neuronal genes. Nature 459, 569-573 (2009).

13. Hogart, A., Wu, D., LaSalle, J. M. \& Schanen, N. C. The comorbidity of autism with the genomic disorders of chromosome 15q11.2-q13. Neurobiol. Dis. 38, 181-191 (2010).

14. Kishino, T., Lalande, M. \& Wagstaff, J. UBE3A/E6-AP mutations cause Angelman syndrome. Nat. Genet. 15, 70-73 (1997).

15. Matsuura, T. et al. De novo truncating mutations in E6-AP ubiquitin-protein ligase gene (UBE3A) in Angelman syndrome. Nat. Genet. 15, 74-77 (1997)

16. Scheffner, M., Werness, B. A., Huibregtse, J. M., Levine, A. J. \& Howley, P. M. The E6 oncoprotein encoded by human papillomavirus types 16 and 18 promotes the degradation of p53. Cell 63, 1129-1136 (1990).

17. Zhao, B. et al. Orthogonal Ubiquitin Transfer through Engineered E1-E2 Cascades for Protein Ubiquitination. Chem. Biol. 19, 1265-1277 (2012).

18. Liu, X. et al. Orthogonal ubiquitin transfer identifies ubiquitination substrates under differential control by the two ubiquitin activating enzymes. Nat. Commun. 8, 14286 (2017).

19. Lee, I. \& Schindelin, H. Structural insights into E1-catalyzed ubiquitin activation and transfer to conjugating enzymes. Cell 134, 268-278 (2008).

20. Olsen, S. K. \& Lima, C. D. Structure of a ubiquitin E1-E2 complex: insights to E1-E2 thioester transfer. Mol. Cell 49, 884-896 (2013).

21. Chao, G. et al. Isolating and engineering human antibodies using yeast surface display. Nat. Protoc. 1, 755-768 (2006)

22. Huang, L. et al. Structure of an E6AP-UbcH7 complex: insights into ubiquitination by the E2-E3 enzyme cascade. Science 286, 1321-1326 (1999).

23. Tagwerker, C. et al. A tandem affinity tag for two-step purification under fully denaturing conditions: application in ubiquitin profiling and protein complex identification combined with in vivocross-linking. Mol. Cell. Proteomics 5, 737-748 (2006).

24. Jacobson, A. D., MacFadden, A., Wu, Z., Peng, J. \& Liu, C. W. Autoregulation of the $26 \mathrm{~S}$ proteasome by in situ ubiquitination. Mol. Biol. Cell 25, 1824-1835 (2014).

25. Zaaroor-Regev, D. et al. Regulation of the polycomb protein Ring1B by selfubiquitination or by E6-AP may have implications to the pathogenesis of Angelman syndrome. Proc. Natl Acad. Sci. USA 107, 6788-6793 (2010).

26. Kumar, S., Talis, A. L. \& Howley, P. M. Identification of HHR23A as a substrate for E6-associated protein-mediated ubiquitination. J. Biol. Chem. 274, 18785-18792 (1999). 
27. Lee, S. Y. et al. Ube3a, the E3 ubiquitin ligase causing Angelman syndrome and linked to autism, regulates protein homeostasis through the proteasomal shuttle Rpn10. Cell Mol. Life. Sci. 71, 2747-2758 (2014).

28. Mellacheruvu, D. et al. The CRAPome: a contaminant repository for affinity purification-mass spectrometry data. Nat. Methods 10, 730-736 (2013).

29. Schwanhausser, B. et al. Global quantification of mammalian gene expression control. Nature 473, 337-342 (2011).

30. Fujioka, A. et al. Dynamics of the Ras/ERK MAPK cascade as monitored by fluorescent probes. J. Biol. Chem. 281, 8917-8926 (2006).

31. Merbl, Y. \& Kirschner, M. W. Large-scale detection of ubiquitination substrates using cell extracts and protein microarrays. Proc. Natl Acad. Sci. USA 106, 2543-2548 (2009).

32. Shimoji, T. et al. Identification of annexin A1 as a novel substrate for E6APmediated ubiquitylation. J. Cell Biochem. 106, 1123-1135 (2009).

33. Tan, M. K., Lim, H. J., Bennett, E. J., Shi, Y. \& Harper, J. W. Parallel SCF adaptor capture proteomics reveals a role for SCFFBXL17 in NRF2 activation via BACH1 repressor turnover. Mol. Cell 52, 9-24 (2013).

34. Pierce, N. W., Kleiger, G., Shan, S. O. \& Deshaies, R. J. Detection of sequential polyubiquitylation on a millisecond timescale. Nature 462, 615-619 (2009).

35. Emanuele, M. J. et al. Global identification of modular cullin-RING ligase substrates. Cell 147, 459-474 (2011).

36. Yen, H. C. \& Elledge, S. J. Identification of SCF ubiquitin ligase substrates by global protein stability profiling. Science 322, 923-929 (2008).

37. Yen, H. C., Xu, Q., Chou, D. M., Zhao, Z. \& Elledge, S. J. Global protein stability profiling in mammalian cells. Science 322, 918-923 (2008).

38. Xu, G. \& Jaffrey, S. R. The new landscape of protein ubiquitination. Nat. Biotechnol. 29, 1098-1100 (2011).

39. Kim, W. et al. Systematic and quantitative assessment of the ubiquitin-modified proteome. Mol. Cell 44, 325-340 (2011).

40. Sarraf, S. A. et al. Landscape of the PARKIN-dependent ubiquitylome in response to mitochondrial depolarization. Nature 496, 372-376 (2013).

41. O'Connor, H. F. et al. Ubiquitin-Activated Interaction Traps (UBAITs) identify E3 ligase binding partners. EMBO Rep. 16, 1699-1712 (2015).

42. Mark, K. G., Loveless, T. B. \& Toczyski, D. P. Isolation of ubiquitinated substrates by tandem affinity purification of E3 ligase-polyubiquitin-binding domain fusions (ligase traps). Nat. Protoc. 11, 291-301 (2016).

43. Mark, K. G., Simonetta, M., Maiolica, A., Seller, C. A. \& Toczyski, D. P. Ubiquitin ligase trapping identifies an $\mathrm{SCF}(\mathrm{Saf} 1)$ pathway targeting unprocessed vacuolar/lysosomal proteins. Mol. Cell 53, 148-161 (2014).

44. Zhuang, M., Guan, S., Wang, H., Burlingame, A. L. \& Wells, J. A. Substrates of IAP ubiquitin ligases identified with a designed orthogonal E3 ligase, the NEDDylator. Mol. Cell 49, 273-282 (2013).

45. Kuslansky, Y. et al. Ubiquitin ligase E6AP mediates nonproteolytic polyubiquitylation of beta-catenin independent of the E6 oncoprotein. J. Gen. Virol. 97, 3313-3330 (2016).

46. Jang, K. W. et al. Ubiquitin ligase CHIP induces TRAF2 proteasomal degradation and NF-kappaB inactivation to regulate breast cancer cell invasion. J. Cell Biochem. 112, 3612-3620 (2011).

47. Zuin, A. et al. Rpn10 monoubiquitination orchestrates the association of the ubiquilin-type DSK2 receptor with the proteasome. Biochem. J. 472, 353-365 (2015).

48. Altun, M. et al. Muscle wasting in aged, sarcopenic rats is associated with enhanced activity of the ubiquitin proteasome pathway. J. Biol. Chem. 285 , 39597-39608 (2010).

49. Kamadurai, H. B. et al. Insights into ubiquitin transfer cascades from a structure of a UbcH5B approximately ubiquitin-HECT(NEDD4L) complex. Mol. Cell 36, 1095-1102 (2009).

50. Kim, H. C., Steffen, A. M., Oldham, M. L., Chen, J. \& Huibregtse, J. M. Structure and function of a HECT domain ubiquitin-binding site. EMBO Rep. 12, 334-341 (2011).

51. Maspero, E. et al. Structure of a ubiquitin-loaded HECT ligase reveals the molecular basis for catalytic priming. Nat. Struct. Mol. Biol. 20, 696-701 (2013).

52. Ogunjimi, A. A. et al. Regulation of Smurf2 ubiquitin ligase activity by anchoring the E2 to the HECT domain. Mol. Cell 19, 297-308 (2005).

53. Cong, L. et al. Multiplex genome engineering using CRISPR/Cas systems. Science 339, 819-823 (2013)

54. Olzmann, J. A., Richter, C. M. \& Kopito, R. R. Spatial regulation of UBXD8 and p97/VCP controls ATGL-mediated lipid droplet turnover. Proc. Natl Acad. Sci. USA 110, 1345-1350 (2013).

55. Pal, P. et al. E3 ubiquitin ligase E6AP negatively regulates adipogenesis by downregulating proadipogenic factor C/EBPalpha. PLoS ONE 8, e65330 (2013).

56. Wolyniec, K. et al. E6AP ubiquitin ligase regulates PML-induced senescence in Myc-driven lymphomagenesis. Blood 120, 822-832 (2012).

57. Levav-Cohen, Y. et al. E6AP is required for replicative and oncogene-induced senescence in mouse embryo fibroblasts. Oncogene 31, 2199-2209 (2012).

58. Wolyniec, K., Levav-Cohen, Y., Jiang, Y. H., Haupt, S. \& Haupt, Y. The E6AP E3 ubiquitin ligase regulates the cellular response to oxidative stress. Oncogene 32, 3510-3519 (2013).
59. Stopa, N., Krebs, J. E. \& Shechter, D. The PRMT5 arginine methyltransferase: many roles in development, cancer and beyond. Cell Mol. Life Sci. 72, 2041-2059 (2015).

60. Rangasamy, S., D’Mello, S. R. \& Narayanan, V. Epigenetics, autism spectrum, and neurodevelopmental disorders. Neurotherapeutics 10, 742-756 (2013).

61. Lv, J., Xin, Y., Zhou, W. \& Qiu, Z. The epigenetic switches for neural development and psychiatric disorders. J. Genet. Genomics 40, 339-346 (2013).

62. Copeland, R. A. Molecular pathways: protein methyltransferases in cancer. Clin. Cancer Res. 19, 6344-6350 (2013).

63. Beaudenon, S. \& Huibregtse, J. M. High-level expression and purification of recombinant E1 enzyme. Methods Enzymol. 398, 3-8 (2005).

64. French, M. E., Kretzmann, B. R. \& Hicke, L. Regulation of the RSP5 ubiquitin ligase by an intrinsic ubiquitin-binding site. J. Biol. Chem. 284, 12071-12079 (2009).

65. Yin, J., Lin, A. J., Golan, D. E. \& Walsh, C. T. Site-specific protein labeling by Sfp phosphopantetheinyl transferase. Nat. Protoc. 1, 280-285 (2006).

66. Gietz, R. D. \& Woods, R. A. Transformation of yeast by lithium acetate/singlestranded carrier DNA/polyethylene glycol method. Methods Enzymol. 350, 87-96 (2002)

67. Gietz, R. D. \& Schiestl, R. H. Applications of high efficiency lithium acetate transformation of intact yeast cells using single-stranded nucleic acids as carrier. Yeast 7, 253-263 (1991).

68. Cutler, A. A. et al. Biochemical isolation of myonuclei employed to define changes to the myonuclear proteome that occur with aging. Aging Cell 16, 738-749 (2017)

69. Comstra, H. S. et al. The interactome of the copper transporter ATP7A belongs to a network of neurodevelopmental and neurodegeneration factors. Elife 6 e24722 (2017).

70. Kall, L., Canterbury, J. D., Weston, J., Noble, W. S. \& MacCoss, M. J. Semisupervised learning for peptide identification from shotgun proteomics datasets. Nat. Methods 4, 923-925 (2007).

\section{Acknowledgements}

We thank Wade Harper, Jon M. Huibregtse, Linda Hicke, and K. Dane Wittrup for providing the gene constructs of Uba1, E6AP, Rsp5, and yeast display vector. This work is supported by grants provided from the National Institutes of Health (GM104498 to J. Y. and H.K.; CA112282 to H.K.), the National Science Foundation (1420193 and 1710460 to J.Y.), the Chicago Biomedical Consortium (Catalyst-026 to H.K. and J.Y., PDR-010 to X.L.), Project 985 startup grant (WF220417001 and WF114117001/004 to B. Z.), the Lynn Sage Breast Cancer Research Foundation, and the Department of Pharmacology at Northwestern University.

\section{Author contributions}

J.Y. and H.K. conceived the idea, Y.W. conducted most experiments, X.L. developed the scheme for cell line construction, tandem affinity purification and target validation, L.Z. contributed to substrate verification, D.D. performed proteomic analysis, K.B., B.Z., and R.L. contributed to cloning and protein ubiquitination assays, H.Z. synthesized biotinxUB, Y.B. performed bioinformatics analysis, J.Y. wrote the manuscript with Y.W., X.L. and H.K., and all authors approved the final version.

\section{Additional information}

Supplementary Information accompanies this paper at 10.1038/s41467-017-01974-7.

Competing interests: The authors declare no competing financial interests.

Reprints and permission information is available online at http://npg.nature.com/ reprintsandpermissions/

Publisher's note: Springer Nature remains neutral with regard to jurisdictional claims in published maps and institutional affiliations.

\footnotetext{
Open Access This article is licensed under a Creative Commons Attribution 4.0 International License, which permits use, sharing, adaptation, distribution and reproduction in any medium or format, as long as you give appropriate credit to the original author(s) and the source, provide a link to the Creative Commons license, and indicate if changes were made. The images or other third party material in this article are included in the article's Creative Commons license, unless indicated otherwise in a credit line to the material. If material is not included in the article's Creative Commons license and your intended use is not permitted by statutory regulation or exceeds the permitted use, you will need to obtain permission directly from the copyright holder. To view a copy of this license, visit http://creativecommons.org/ licenses/by/4.0/.
}

(C) The Author(s) 2017 